\title{
Relative Hecke's integral formula for an arbitrary extension of number fields
}

\author{
Hohto Bekki
}

\begin{abstract}
In this article, we present a generalized Hecke's integral formula for an arbitrary extension $E / F$ of number fields. As an application, we present relative versions of the residue formula and Kronecker's limit formula for the "relative" partial zeta function of $E / F$. This gives a simultaneous generalization of two different known results given by Hecke himself and Yamamoto.
\end{abstract}

\section{Introduction}

Let $k$ be a number field (of finite degree), and $\mathcal{O}_{k}$ be the ring of integers of $k$. Let $\mathscr{A}$ be an ideal class of $k$, and let $\mathfrak{a} \in \mathscr{A}$. Then the partial zeta function $\zeta_{k}(\mathscr{A}, s)=\zeta_{k}(\mathfrak{a}, s)$ associated to the ideal class $\mathscr{A}$ (or to the ideal $\mathfrak{a}$ ) is defined as,

$$
\zeta_{k}(\mathscr{A}, s):=\sum_{\substack{\mathfrak{b} \in \mathcal{A}^{\prime} \\ \mathfrak{b} \subset \mathcal{O}_{k}}} \frac{1}{N \mathfrak{b}^{s}} \quad(\operatorname{Re}(s)>1) .
$$

In the case where $k$ is real quadratic, the classical Hecke's integral formula expresses this partial zeta function as the integral of the real analytic Eisenstein series along the closed geodesic on the modular curve $S L_{2}(\mathbb{Z}) \backslash \mathfrak{h}$ associated to the ideal class $\mathscr{A}$, where $\mathfrak{h}:=\{z \in \mathbb{C} \mid \operatorname{Im}(z)>0\}$ is the Poincaré upper half plane.

To be precise, let $k$ be real quadratic, and fix an embedding $k \hookrightarrow \mathbb{R}$. Suppose $\mathfrak{a} \in \mathscr{A}$ is taken to be of the form $\mathfrak{a}=\mathbb{Z}+\mathbb{Z} \alpha \subset k$, where $\alpha \in k$ is a real quadratic irrational. Let $\bar{\alpha}$ be the conjugate of $\alpha$ over $\mathbb{Q}$, and let $\varpi$ be the geodesic on $\mathfrak{h}$ connecting $\alpha$ and $\bar{\alpha}$ :

$$
\varpi: \mathbb{R}_{>0} \rightarrow \mathfrak{h} ; t \mapsto \frac{\alpha t i+\bar{\alpha} t^{-1}}{t i+t^{-1}}
$$

Then it is known that $\varpi$ projected to $S L_{2}(\mathbb{Z}) \backslash \mathfrak{h}$ becomes periodic. More precisely, $\varpi$ induces a closed geodesic

$$
\bar{\varpi}: \mathbb{R}_{>0} / \varepsilon^{2 \mathbb{Z}} \rightarrow S L_{2}(\mathbb{Z}) \backslash \mathfrak{h},
$$

where $\varepsilon>1$ is a fundamental unit of $\mathcal{O}_{k}$ (cf. [2], [15]).

Now, let $E(z, s)$ be the real analytic Eisenstein series defined by

$$
E(z, s):=\frac{1}{2} \sum_{\substack{(c, d) \in \mathbb{Z}^{2} \\(c, d)=1}} \frac{\operatorname{Im}(z)^{s}}{|c z+d|^{2 s}}, \quad \text { for } z \in \mathfrak{h} \text { and } s \in \mathbb{C}, \operatorname{Re}(s)>1
$$


which is well-defined on $S L_{2}(\mathbb{Z}) \backslash \mathfrak{h}$. Then we have the following Hecke's integral formula.

Theorem 1.0.1 ([10]). We have

$$
\int_{\mathbb{R}_{>0} / \varepsilon^{2 \mathbb{Z}}} E(\bar{\varpi}(t), s) \frac{d t}{t}=\frac{1}{2} d_{k}^{s / 2} \frac{\Gamma(s / 2)^{2}}{\Gamma(s)} \frac{\zeta_{k}\left(\mathfrak{a}^{-1}, s\right)}{\zeta_{\mathbb{Q}}(2 s)},
$$

where $d_{k}$ is the discriminant of $k$.

Many authors including Hecke himself have studied generalizations of this formula. Hecke [10] generalizes the formula to the case of an arbitrary number field, and HiroeOda [11] extend Hecke's result to $L$-functions twisted by Grossencharacters. Another generalization is obtained by Yamamoto [18], who generalizes the formula to the case of an arbitrary quadratic extension $E / F$ of number fields. In the following we refer to any generalization of Theorem 1.0.1 as Hecke's integral formula.

In a previous paper [2], motivated by the analogy between the above periodicity of the geodesic $\varpi$ and the classical Lagrange's theorem in the theory of continued fractions, we have considered generalization of closed geodesics in the symmetric space for $G L_{n}$. As a result, we have established some new geodesic multi-dimensional continued fraction algorithms, and have proved generalizations of Lagrange's theorem. In this paper, using the same idea as in [2], we present Hecke's integral formula for an arbitrary extension $E / F$ of number fields (Theorem 3.3.3). Hecke's result corresponds to the case where $F=\mathbb{Q}$, and Yamamoto's result corresponds to the case where $[E: F]=2$. In our argument, we are naturally led to introduce a "relative" partial zeta function $\zeta_{E / F, \mathscr{A}}(A, s)$ $\left(\mathscr{A} \in C l_{F}, A \in C l_{E}\right)$ (Definition 3.3.2), which gives a "decomposition of the partial zeta function $\zeta_{E}(A, s)$ along $C l_{F}$ ":

$$
\zeta_{E}(A, s)=\sum_{\mathscr{A} \in C l_{F}} \zeta_{E / F, \mathscr{A}}(A, s)
$$

As an application of our Hecke's integral formula, we obtain the residue formula and Kronecker's limit formula for this relative partial zeta function $\zeta_{E / F, \mathscr{A}}(A, s)$, that is, formulas for the residue and the constant term of $\zeta_{E / F, \mathscr{A}}(A, s)$ at $s=1$ (Theorem 5.3.1). The author thinks it is interesting that both the special value of $\zeta_{E}(A, s)$ at $s=1$ and the special value of $\zeta_{F}\left(\mathscr{A}^{-1}, s\right)$ at $s=\boldsymbol{n}$ appear simultaneously in the residue formula (5.13) of the relative partial zeta function $\zeta_{E / F, \mathscr{A}}(A, s)$. As far as the author is aware, such a phenomenon has not been observed in the previous works.

There are also many preceding works on Kronecker's limit formula for the zeta functions of number fields. Hecke remarks in [10] that one can deduce Kronecker's limit formula for general number fields from the result of Epstein [7, p. 644]. Liu and Masri [14] use this formula for totally real fields to obtain an analogue of Kronecker's solution of Pell's equation. Bump and Goldfeld [5] give a different proof in the case of totally real cubic fields. The case of relative quadratic extensions of number fields is obtained by Yamamoto [18], and our result generalizes all of these results. 
Outline of this paper In Section 2, we set up generalizations of the Poincaré upper half plane $\mathfrak{h}$, the above geodesic $\varpi$ on $\mathfrak{h}$, and the real analytic Eisenstein series $E(z, s)$. We use the symmetric space for $\operatorname{Res}_{F / \mathbb{Q}} G L_{n}$ as a generalization of $\mathfrak{h}$ where $F$ is a number field (cf. Borel [3]), and define a certain totally geodesic submanifold called the Heegner object in the symmetric space which plays a part of the above geodesic $\varpi$. Then we consider the Eisenstein series for $\operatorname{Res}_{F / \mathbb{Q}} G L_{n}$ following the general construction of Langlands [13. In Section 3, we prove our first main theorem: Theorem 3.3.3.

Sections 4 and 5 are devoted to prove the residue formula and Kronecker's limit formula for the relative partial zeta function $\zeta_{E / F, \mathscr{A}}(A, s)$. In Section 4, we compute the Fourier expansion of the Eisenstein series as a preparation. In Section 5, we first prove the residue formula and Kronecker's limit formula for the Eisenstein series using the Fourier expansion. Then using Theorem 3.3.3, we obtain our second main theorem: Theorem 5.3.1.

Some remarks on Eisenstein series In this paper, we construct Eisenstein series by generalizing the argument of Goldfeld [8]. This construction fits into the general theory of Eisenstein series established by Langlands [13]. Therefore some of the results in this paper concerning the Eisenstein series (e.g., the convergence, analytic continuation, and some part of Fourier coefficients) might be obtained directly from the general theory. However, as far as the author is aware, our Eisenstein series $E_{L, \mathscr{A}}(z, s)\left(\mathscr{A} \in C l_{F}\right)$ (Definition 2.2.7) has not been studied well, while the sum $E_{L}(z, s)=\sum_{\mathscr{A} \in C l_{F}} E_{L, \mathscr{A}}(z, s)$ is a generalization of those traditional Eisenstein series studied by Asai [1], JorgensonLang [12, Yoshida [19], and Yamamoto [18] in the case where $n=2$. In Sections 4 and 5, we give an explicit Fourier expansion formula, residue formula, and Kronecker's limit formula for our Eisenstein series $E_{L, \mathscr{A}}(z, s)$, which gives a generalization of the corresponding results proved in [1], [12], [18] and [14].

\subsection{Convention}

Let $\Lambda$ be any index set, and let $\left\{X_{\lambda}\right\}_{\lambda \in \Lambda}$ be any family of sets indexed by $\Lambda$. For $x \in \prod_{\lambda \in \Lambda} X_{\lambda}$, we often denote by $x_{\lambda} \in X_{\lambda}$ the $\lambda$-component of $x$ without specifying.

In this paper, a number field is always assumed to be of finite degree over the field $\mathbb{Q}$ of rational numbers. For a number field $k$, we denote by $S_{k}$ the set of archimedean places of $k$. For $\sigma \in S_{k}$, we denote by $k_{\sigma}$ the completion of $k$ at $\sigma$, and denote by $n_{\sigma}:=\left[k_{\sigma}: \mathbb{R}\right]$ the local extension degree. We also denote by $\sigma: k \hookrightarrow k_{\sigma}$ the completion map. We denote by $k_{\infty}:=k \otimes_{\mathbb{Q}} \mathbb{R}$ the infinite adele of $k$. Then we have $k_{\infty} \simeq \prod_{\sigma \in S_{k}} k_{\sigma}$. The number of archimedean (resp. real, complex) places is denoted by $r_{k}\left(\right.$ resp. $r_{1}(k), r_{2}(k)$ ). As usual, we denote by $\mathcal{O}_{k}$ the ring of integers, $\mathcal{O}_{k}^{\times}$its group of units, $C l_{k}$ the ideal class group, and $h_{k}$ the class number. We denote by $d_{k} \in \mathbb{Z}$ the absolute discriminant and by $\mathfrak{d}_{k}$ the different ideal. For a fractional $\mathcal{O}_{k}$-ideal $\mathfrak{a} \subset k$, we denote by $[\mathfrak{a}] \in C l_{k}$ the ideal class of $\mathfrak{a}$. 
We equip $\mathbb{C}\left(\right.$ resp. $\left.\mathbb{C}^{n}\right)$ with the following normalized absolute value | | (resp. || ||),

$$
\begin{aligned}
& ||: \mathbb{C} \rightarrow \mathbb{R}_{\geq 0} ; x+i y \mapsto\left(x^{2}+y^{2}\right)^{1 / 2} \quad(x, y \in \mathbb{R}), \\
& \|\|: \mathbb{C}^{n} \rightarrow \mathbb{R}_{\geq 0} ;\left(x_{1}, \ldots, x_{n}\right) \mapsto\left(\left|x_{1}\right|^{2}+\cdots+\left|x_{n}\right|^{2}\right)^{1 / 2} .
\end{aligned}
$$

For $\sigma \in S_{k}$, let us choose an embedding $k_{\sigma} \hookrightarrow \mathbb{C}$. Then the above absolute value induces an absolute value || (resp. \|\|$)$ on $k_{\sigma}\left(\right.$ resp. $\left.k_{\sigma}^{n}\right)$. This is clearly independent of the chosen embedding. For $r \geq 1$, we define the pairing $\langle\rangle:, k_{\infty}^{r} \times k_{\infty}^{r} \rightarrow \mathbb{R}$ by

$$
\left\langle\left(x_{1}, \ldots, x_{r}\right),\left(y_{1}, \ldots, y_{r}\right)\right\rangle:=\sum_{i=1}^{r} \operatorname{Tr}_{k / \mathbb{Q}}\left(x_{i} y_{i}\right), \quad\left(x_{i}, y_{i} \in k_{\infty}\right) .
$$

Here $\operatorname{Tr}_{k / \mathbb{Q}}: k_{\infty} \rightarrow \mathbb{R}$ is the field trace map naturally extended to $k_{\infty}$.

Suppose now $k^{\prime} / k$ is an extension of number fields. For $\sigma \in S_{k}$, we denote by $S_{k^{\prime}, \sigma}$ the set of places of $k^{\prime}$ above $\sigma$. Then for $\sigma \in S_{k}$ and $\tau \in S_{k^{\prime}, \sigma}$, we fix an embedding $k_{\sigma} \subset k_{\tau}^{\prime}$, and denote by $n_{\tau \mid \sigma}:=\left[k_{\tau}^{\prime}: k_{\sigma}\right]$ the degree of the local filed extension.

In the following, we basically fix an extension $E / F$ of number fields in our argument. However, when we introduce general notation, we use $k$ or $k^{\prime} / k$ to represent an arbitrary number field or extension of number fields.

\section{Generalized upper half space over $F$}

In order to generalize closed geodesics on the modular curve $S L_{2}(\mathbb{Z}) \backslash \mathfrak{h}$, first observe that the geodesic $\varpi$ (in Section 1) on $\mathfrak{h}$ connecting conjugate real quadratic irrationals $\alpha$ and $\bar{\alpha}$ is obtained by the left translation of the imaginary axis $I:=i \mathbb{R}_{>0} \subset \mathfrak{h}$ by the matrix $W=\left(\begin{array}{cc}\alpha & \bar{\alpha} \\ 1 & 1\end{array}\right) \in G L_{2}(\mathbb{R})$. See Figure 1 below.

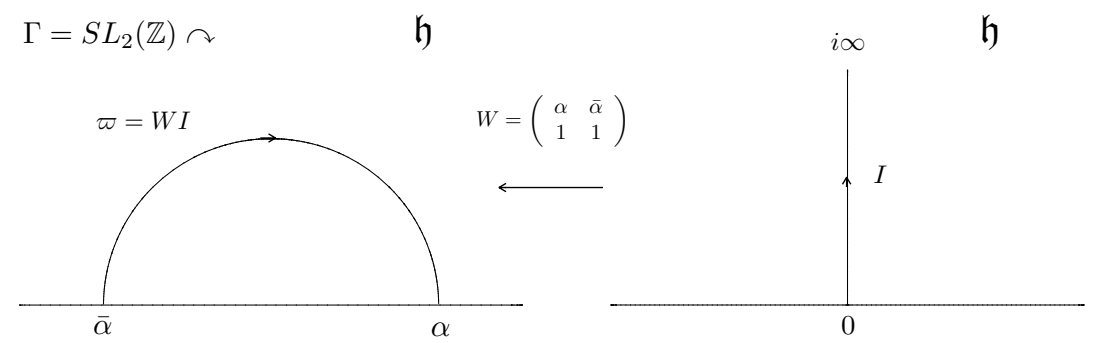

Figure 1: The geodesic $\varpi$

In the following, we generalize each of these objects $\mathfrak{h}, W, I, \varpi$, and $S L_{2}(\mathbb{Z})$.

Let $F$ be a number field of degree $d$. We fix an embedding $F_{\sigma} \subset \mathbb{C}$ for each $\sigma \in$ $S_{F}$. Let us consider an algebraic group $G:=\operatorname{Res}_{F / \mathbb{Q}} G L_{n}$, where $\operatorname{Res}_{F / \mathbb{Q}}$ is the Weil 
restriction. Then we take a standard maximal compact subgroup $K=\prod_{\sigma \in S_{F}} K_{\sigma}$ of $G(\mathbb{R}) \simeq \prod_{\sigma \in S_{F}} G L_{n}\left(F_{\sigma}\right)$ as follows:

$$
\begin{gathered}
K_{\sigma}:=O(n) \subset G L_{n}\left(F_{\sigma}\right)=G L_{n}(\mathbb{R}), \quad \text { if } \sigma \text { is real, } \\
K_{\sigma}:=U(n) \subset G L_{n}\left(F_{\sigma}\right)=G L_{n}(\mathbb{C}), \quad \text { if } \sigma \text { is complex. }
\end{gathered}
$$

Definition 2.0.1 (Generalized upper half space over $F$, Cf. [3], [8]). Set

$$
\mathfrak{h}_{\sigma}^{n}:=G L_{n}\left(F_{\sigma}\right) / F_{\sigma}^{\times} K_{\sigma}, \quad \text { for } \sigma \in S_{F} .
$$

Then we define the generalized upper half space for $G$ to be the symmetric space

$$
\mathfrak{h}_{F}^{n}:=G(\mathbb{R}) / F_{\infty}^{\times} K \simeq \prod_{\sigma \in S_{F}} \mathfrak{h}_{\sigma}^{n} .
$$

In the following, for $g \in G(\mathbb{R})=\prod_{\sigma \in S_{F}} G L_{n}\left(F_{\sigma}\right)$, we always denote by $g_{\sigma}$ the $\sigma$ component of $g$, and denote by $[g] \in \mathfrak{h}_{F}^{n}$ the class represented by $g$.

Remark. In the case where $F=\mathbb{Q}, n=2$, we have an isometry $\mathfrak{h}_{\mathbb{Q}}^{2} \stackrel{\sim}{\rightarrow} \mathfrak{h} ;[g] \mapsto g i$, where the action of $g$ on $i \in \mathfrak{h}$ is the usual linear fractional transformation.

\subsection{Heegner objects}

In this section, we define a certain totally geodesic submanifold of $\mathfrak{h}_{F}^{n}$ called the Heegner object. We closely follow the construction in [2, Section 2], but have slightly modified the argument in order to deal with the ideal class group of $F$.

Let $F$ be the same as above, and let $E / F$ be a field extension of degree $n$. For $\sigma \in S_{F}$ and $\tau \in S_{E, \sigma}$, we fix an embedding $E_{\tau} \subset \mathbb{C}$ so that $F_{\sigma} \subset E_{\tau} \subset \mathbb{C}$. Let us fix a basis $w_{1}, \ldots, w_{n} \in E$ of $E$ over $F$. Set $w:={ }^{t}\left(w_{1} \cdots w_{n}\right) \in E^{n}$. First, we define two isomorphisms $E_{\infty} \simeq F_{\infty}^{n}$ of $F_{\infty}$-modules using local and global data on $E_{\infty}$.

local: For $\sigma \in S_{F}$ and $\tau \in S_{E, \sigma}$, we fix an isomorphism $E_{\tau} \simeq F_{\sigma}^{n_{\tau \mid \sigma}}$ of $F_{\sigma}$-vector spaces as follows: If $n_{\tau \mid \sigma}=1$, then simply $E_{\tau}=F_{\sigma}$. Otherwise, $\sigma$ is real and $\tau$ is complex, and $n_{\tau \mid \sigma}=2$. Therefore using $\mathbb{C} \simeq \mathbb{R}^{2} ; x+i y \mapsto(y, x)$, we obtain $E_{\tau}=\mathbb{C} \simeq \mathbb{R}^{2}=F_{\sigma}^{2}$. This induces

$$
E_{\sigma}:=E \otimes_{F} F_{\sigma} \simeq \prod_{\tau \mid \sigma} E_{\tau} \simeq \prod_{\tau \mid \sigma} F_{\sigma}^{n_{\tau \mid \sigma}} \simeq F_{\sigma}^{n}
$$

and by taking the product over $\sigma \in S_{F}$, we get

$$
\iota: E_{\infty} \stackrel{\sim}{\rightarrow} F_{\infty}^{n} .
$$

global: Since $w_{1}, \ldots, w_{n}$ is a basis of $E$ over $F$, we have an isomorphism $w: F^{n} \stackrel{\sim}{\rightarrow}$ $E ; \overline{x \mapsto x} \cdot w$, of $F$-vector spaces. Here we regard $x$ as a row vector, and $x \cdot w$ is the scalar product. Thus by tensoring $\mathbb{R}$ over $\mathbb{Q}$, we obtain

$$
w: F_{\infty}^{n} \stackrel{\sim}{\rightarrow} E_{\infty} .
$$


Then we define $W \in G(\mathbb{R})$ so that $W=\iota \circ w$ in $\operatorname{End}\left(F_{\infty}^{n}\right)$, that is,

$$
\iota \circ w: F_{\infty}^{n} \stackrel{\sim}{\rightarrow} E_{\infty} \stackrel{\sim}{\rightarrow} F_{\infty}^{n} ; x \mapsto x W .
$$

Next, we generalize $I$. For a number field $k$, we set $T_{k}:=\prod_{\sigma \in S_{k}} \mathbb{R}_{>0}$. Then $T_{k}$ acts naturally on $k_{\infty} \simeq \prod_{\sigma \in S_{k}} k_{\sigma}$ by the component-wise multiplication. For an extension $k^{\prime} / k$ of number fields, the field norm map $N_{k^{\prime} / k}: k^{\prime} \rightarrow k$ induces a homomorphism,

$$
N_{k^{\prime} / k}: T_{k^{\prime}} \rightarrow T_{k} ;\left(t_{\tau}\right)_{\tau \in S_{k^{\prime}}} \mapsto\left(\prod_{\tau \mid \sigma} t_{\tau}^{n_{\tau \mid \sigma}}\right)_{\sigma \in S_{k}}
$$

We denote by $T_{k^{\prime} / k}:=\operatorname{ker}\left(T_{k^{\prime}} \stackrel{N_{k^{\prime} / k}}{\rightarrow} T_{k}\right)$ the kernel of this norm homomorphism.

Then the action of $T_{E / F}$ on $F_{\infty}^{n}$ via $E_{\infty} \stackrel{\iota}{\simeq} F_{\infty}^{n}$, which is clearly as $F_{\infty}$-module, induces a group homomorphism

$$
I=I_{E / F}: T_{E / F} \rightarrow G(\mathbb{R})=G L_{n}\left(F_{\infty}\right) .
$$

Definition 2.1.1 (Cf. [2]). We define the Heegner object associated to the basis $w$ of $E$ over $F$ by

$$
\varpi=\varpi_{w}: T_{E / F} \rightarrow \mathfrak{h}_{F}^{n} ; t \mapsto[W I(t)],
$$

where $[W I(t)]$ denotes the class of $W I(t)$ in $\mathfrak{h}_{F}^{n}$ as remarked before.

Remark. If $F=\mathbb{Q}$ and $E$ is imaginary quadratic, then the image of $\varpi_{w}$ is just a Heegner point on $\mathfrak{h}=\mathfrak{h}_{\mathbb{Q}}^{2}$.

Arithmetic subgroup and periodicity We define an arithmetic subgroup $\Gamma$ of $G(\mathbb{R})$ and discuss the periodicity of the Heegner object $\varpi$ with respect to $\Gamma$.

Let $L \subset F^{n}$ be an $\mathcal{O}_{F}$-lattice, that is, an $\mathcal{O}_{F}$-submodule such that $L \otimes_{\mathcal{O}_{F}} F=F^{n}$. Consider the natural right action of $S L_{n}(F)$ on the space $F^{n}$ of row vectors. Define

$$
\Gamma_{L}:=\operatorname{Stab}_{S L_{n}(F)}(L)=\left\{\gamma \in S L_{n}(F) \mid L \gamma=L\right\},
$$

where "Stab" is the stabilizer subgroup. Then $\Gamma_{L}$ acts properly discontinuously on $\mathfrak{h}_{F}^{n}$ from the left.

Now let $\mathfrak{A} \subset E$ be a fractional $\mathcal{O}_{E}$-ideal. We take $L \subset F^{n}$ so that $L$ corresponds to $\mathfrak{A}$ under the isomorphism (2.7) (i.e., $w: L \stackrel{\sim}{\rightarrow} \mathfrak{A}$ ), and set

$$
\Gamma=\Gamma_{\mathfrak{A}}:=\Gamma_{L}
$$

For a number field $k$, we denote by $U_{k}$ the image of the unit group $\mathcal{O}_{k}^{\times}$under the following "multiplicative" regulator map:

$$
\operatorname{reg}_{k}^{\times}: \mathcal{O}_{k}^{\times} \rightarrow T_{k} ; u \mapsto(|\sigma(u)|)_{\sigma \in S_{k}} .
$$

Then, by Dirichlet's unit theorem, $U_{k}$ is a lattice in $T_{k / \mathbb{Q}} \subset T_{k}$, that is, a discrete cocompact subgroup of $T_{k / \mathbb{Q}}$. 
Now, for an extension $k^{\prime} / k$ of number fields, let $\mathcal{O}_{k^{\prime} / k}^{\times}:=\operatorname{ker}\left(\mathcal{O}_{k^{\prime}}^{\times} \stackrel{N_{k^{\prime} / k}}{\rightarrow} \mathcal{O}_{k}^{\times}\right)$be the relative unit group of $k^{\prime} / k$. We denote by $U_{k^{\prime} / k}$ the image of $\mathcal{O}_{k^{\prime} / k}^{\times}$under the following relative regulator map $\operatorname{reg}_{k^{\prime} / k}^{\times}$:

$$
\operatorname{reg}_{k^{\prime} / k}^{\times}:=\left.\operatorname{reg}_{k^{\prime}}^{\times}\right|_{\mathcal{O}_{k^{\prime} / k}^{\times}}: \mathcal{O}_{k^{\prime} / k}^{\times} \rightarrow T_{k^{\prime} / k},
$$

which is just the restriction of $\operatorname{reg}_{k^{\prime}}^{\times}$. Then we easily see that $U_{k^{\prime} / k}$ is a lattice in $T_{k^{\prime} / k}$.

Let $E / F$ be as before, and let $\pi: \mathfrak{h}_{F}^{n} \rightarrow \Gamma \backslash \mathfrak{h}_{F}^{n}$ be the natural projection.

Proposition 2.1.2. The map $T_{E / F} \stackrel{\varpi}{\rightarrow} \mathfrak{h}_{F}^{n} \stackrel{\pi}{\rightarrow} \Gamma \backslash \mathfrak{h}_{F}^{n}$ factors through

$$
\bar{\varpi}: T_{E / F} / U_{E / F} \rightarrow \Gamma \backslash \mathfrak{h}_{F}^{n} .
$$

In order to prove this proposition, we prepare a lemma. Let

$$
\varrho_{w}: E^{\times} \rightarrow \operatorname{Aut}_{F}(E) \simeq G L_{n}(F) \subset G(\mathbb{R})
$$

be the regular representation of $E$ over $F$ with respect to the basis $w$ of $E$ over $F$, that is, for any row vector $x \in F^{n}$, we have $x \varrho_{w}(\alpha) w=\alpha x w$ in $E$.

Lemma 2.1.3. For $u \in \mathcal{O}_{E / F}^{\times}$, we have $W^{-1} \varrho_{w}(u) W \equiv I\left(\operatorname{reg}_{E / F}^{\times}(u)\right) \bmod K$.

Proof. Set $g:=W^{-1} \varrho_{w}(u) W$ and $\rho:=\operatorname{reg}_{E / F}^{\times}(u)$. By the definition of $W$ and $\varrho_{w}$, the left hand side $g=W^{-1} \varrho_{w}(u) W$ represents the multiplication by $u$ on $E_{\infty}$ via $E_{\infty} \stackrel{\iota}{\simeq} F_{\infty}^{n}$. Now, for $\tau \in S_{E}$, the multiplication by $\tau(u)$ on $E_{\tau} \subset \mathbb{C}$ decomposes into the scaling by $|\tau(u)|$ and a rotation. Therefore $g$ decomposes as $g=I(\rho) R$ for some $R \in K$.

Proof of Proposition 2.1.2. Let $\rho \in U_{E / F}$. We have to show that there exists $\gamma \in \Gamma$ such that $\varpi(\rho t)=\gamma \varpi(t)$ holds for all $t \in T_{E / F}$. Take any $u \in \mathcal{O}_{E / F}^{\times}$such that $\operatorname{reg}_{E / F}^{\times}(u)=\rho$. Let $\gamma:=\varrho_{w}(u)$. Since $N_{E / F}(u)=1$ and the multiplication by $u$ preserves the ideal $\mathfrak{A}$, we have $\gamma \in \Gamma$. Then, by Lemma 2.1.3, we obtain $\varpi(\rho t)=[W I(\rho t)]=[\gamma W I(t)]=\gamma \varpi(t)$ for all $t \in T_{E / F}$.

\subsection{Langlands Eisenstein series for $\operatorname{Res}_{F / \mathbb{Q}} G L_{n}$}

In this section, we set up basic definitions of Eisenstein series on $\mathfrak{h}_{F}^{n}$. We apply the general construction of the so-called Langlands Eisenstein series to our case $G=\operatorname{Res}_{F / \mathbb{Q}} G L_{n}$. We basically follow the argument in Goldfeld [8]. However, since [8] deals only with the case where $F=\mathbb{Q}$, we need some additional consideration.

In order to define the Langalnds Eisenstein series, we have to choose a parabolic subgroup of $\Gamma_{L}$. Let us denote by $\mathfrak{a} \hookrightarrow L$ a data consisting of

- $L \subset F^{n}$, an $\mathcal{O}_{F}$-latiice (not necessarily defined from the fractional ideal $\mathfrak{A} \subset E$ ),

- $\mathfrak{a} \subset F$, a fractional $\mathcal{O}_{F}$-ideal such that $1 \in \mathfrak{a}$ (we call such $\mathfrak{a}$ an anti-integral ideal), 
- $\mathfrak{a} \hookrightarrow L$, a split injective $\mathcal{O}_{F}$-homomorphism, that is, the exact sequence $0 \rightarrow \mathfrak{a} \hookrightarrow L \rightarrow$ $L / \mathfrak{a} e \rightarrow 0$ splits, where we denote by $e \in L$ the image of $1 \in \mathfrak{a}$.

In the following, we refer to such a data $\mathfrak{a} \hookrightarrow L$ a parabolic data. Set $\Gamma:=\Gamma_{L}$ (cf. (2.12)). Then we define the parabolic subgroup $P_{\mathfrak{a} \hookrightarrow L}$ associated to the data $\mathfrak{a} \hookrightarrow L ; 1 \mapsto e$ as

$$
P=P_{\mathfrak{a} \hookrightarrow L}:=\operatorname{Stab}_{\Gamma_{L}}(\mathfrak{a} \hookrightarrow L)=\left\{\gamma \in \Gamma_{L} \mid \mathfrak{a} e \gamma=\mathfrak{a} e \text { in } L\right\} .
$$

Next, we define a certain left $P$-invariant function on $\mathfrak{h}_{F}^{n}$.

Definition 2.2.1. We define a function Det $=\operatorname{Det}_{\mathfrak{a} \hookrightarrow L}: \mathfrak{h}_{F}^{n} \rightarrow \mathbb{R}_{\geq 0}$ by

$$
\operatorname{Det}([g]):=\prod_{\sigma \in S_{F}} \frac{\left|\operatorname{det} g_{\sigma}\right|^{n_{\sigma}}}{\left\|e g_{\sigma}\right\|^{n n_{\sigma}}}, \quad \text { for } g=\left(g_{\sigma}\right)_{\sigma} \in G(\mathbb{R}),
$$

where \|\| is the normalized absolute value defined as (1.8). Here by an abuse of notation, we denote also by $e$ the image of $e \in L \subset F^{n}$ under the completion map $\sigma: F^{n} \hookrightarrow F_{\sigma}^{n}$, and $e g_{\sigma}$ is an element of $F_{\sigma}^{n}$. It is clear that this function is well-defined on $\mathfrak{h}_{F}^{n}$.

Lemma 2.2.2. The function $\operatorname{Det}(z)$ is left $P$-invariant, that is, $\operatorname{Det}(\gamma z)=\operatorname{Det}(z)$ for all $z \in \mathfrak{h}_{F}^{n}$ and $\gamma \in P$.

Proof. Since $\gamma \in \Gamma_{L}$, we have $\operatorname{det} \gamma=1$. Now, since $\gamma \in P$ preserves a $\subset \subset L, \gamma$ acts on $e$ by the multiplication by some $u \in \mathcal{O}_{F}^{\times}$. Then, $\prod_{\sigma}\left\|e \gamma g_{\sigma}\right\|^{n_{\sigma}}=\prod_{\sigma}\left\|u e g_{\sigma}\right\|^{n_{\sigma}}=$ $\prod_{\sigma}|\sigma(u)|^{n_{\sigma}}\left\|e g_{\sigma}\right\|^{n_{\sigma}}=\prod_{\sigma}\left\|e g_{\sigma}\right\|^{n_{\sigma}}$. This shows the lemma.

Definition 2.2.3. We define the Eisenstein series associated to the data $\mathfrak{a} \hookrightarrow L$ as

$$
E_{\mathfrak{a} \hookrightarrow L}(z, s):=\sum_{\gamma \in P \backslash \Gamma} \operatorname{Det}(\gamma z)^{s}, \quad \text { for } z \in \mathfrak{h}_{F}^{n} \text { and } s \in \mathbb{C}, \operatorname{Re}(s)>1 .
$$

We prove the absolute convergence of $E_{\mathfrak{a} \hookrightarrow L}(z, s)$ in Section 3.2 .

More explicit form In order to study $E_{\mathfrak{a} \hookrightarrow L}(z, s)$, we rewrite the sum more explicitly. For an $\mathcal{O}_{F}$-lattice $L \subset F^{n}$ and a fractional $\mathcal{O}_{F}$-ideal $\mathfrak{a} \subset F$, we define

$$
\begin{aligned}
L_{\mathfrak{a}} & :=\{x \in L-\{0\} \mid F x \cap L=\mathfrak{a} x\} \\
& =\left\{x \in L \mid \mathfrak{a} \rightarrow L ; \alpha \mapsto \alpha x, \text { is a split injective } \mathcal{O}_{F} \text {-homomorphism }\right\} .
\end{aligned}
$$

Lemma 2.2.4. (1) We have a decomposition $L-\{0\}=\coprod_{\mathfrak{a} \text { :anti-int. }} L_{\mathfrak{a}}$, where the union is taken over the anti-integral ideals.

(2) For a parabolic data $\mathfrak{a} \hookrightarrow L ; 1 \mapsto e$, we have the following bijection:

$$
P_{\mathfrak{a} \hookrightarrow L} \backslash \Gamma_{L} \stackrel{\sim}{\longrightarrow} \mathcal{O}_{F}^{\times} \backslash L_{\mathfrak{a}} ; \gamma \mapsto e \gamma .
$$

We need the following structure theorem for finitely generated projective modules over Dedekind domains. See [4, Chapter 7, §4, Proposition 24] for example. 
Proposition 2.2.5. Let $A$ be a Dedekind domain. Then any non-zero finitely generated projective module $M$ over $A$ is isomorphic to $A^{r-1} \oplus \mathfrak{a}$ for some $r \in \mathbb{Z}_{\geq 1}$ and a fractional $A$-ideal $\mathfrak{a}$. Moreover $r$ and the ideal class of $\mathfrak{a}$ in this presentation are unique.

Proof of Lemma 2.2.4. (1) is clear. To see (2), it suffices to show that the right (matrix) action of $\Gamma_{L}$ on $\mathcal{O}_{F}^{\times} \backslash L_{\mathfrak{a}}$ is transitive. Take any $x \in L_{\mathfrak{a}}$. Then by (2.22), there exist isomorphisms $\varphi_{1}: \mathfrak{a} \oplus L / \mathfrak{a} e \stackrel{\sim}{\rightarrow} L$ which sends $(1,0)$ to $e$, and $\varphi_{2}: \mathfrak{a} \oplus L / \mathfrak{a} x \stackrel{\sim}{\rightarrow} L$ which sends $(1,0)$ to $x$. Then by Proposition 2.2.5, we see that there exists an $\mathcal{O}_{F}$-isomorphism $\varphi: L / \mathfrak{a} e \stackrel{\sim}{\rightarrow} L / \mathfrak{a} x$. Then for any $u \in \mathcal{O}_{F}^{\times}$, we obtain an automorphism

$$
L \stackrel{\varphi_{1}^{-1}}{\longrightarrow} \mathfrak{a} \oplus L / \mathfrak{a} e \stackrel{\times u \oplus \varphi}{\sim} \mathfrak{\longrightarrow} \oplus L / \mathfrak{a} x \stackrel{\varphi_{2}}{\longrightarrow} L
$$

of $L$ which sends $e$ to $x$. This extends to $\gamma \in G L_{n}(F)$ such that $\operatorname{det} \gamma \in \mathcal{O}_{F}^{\times}$. By replacing $u$ with $u(\operatorname{det} \gamma)^{-1}$, we obtain $\gamma \in \Gamma_{L}$ such that $e \gamma \equiv x \bmod \mathcal{O}_{F}^{\times}$.

Corollary 2.2.6. We can rewrite the sum (2.20) as

$$
E_{\mathfrak{a} \hookrightarrow L}([g], s)=\sum_{x \in \mathcal{O}_{F}^{\times} \backslash L_{\mathfrak{a}}} \prod_{\sigma \in S_{F}} \frac{\left|\operatorname{det} g_{\sigma}\right|^{n_{\sigma} s}}{\|\left. x g_{\sigma}\right|^{\mid n n_{\sigma} s}}, \quad \text { for } g \in G(\mathbb{R}) .
$$

In particular, $E_{\mathfrak{a} \hookrightarrow L}(z, s)$ depends only on the lattice $L$ and the anti-integral ideal $\mathfrak{a}$.

We define some variants of $E_{\mathfrak{a} \hookrightarrow L}(z, s)$.

Definition 2.2.7. Using the expression (2.25), for $\mathscr{A} \in C l_{F}$, we define

$$
E_{L, \mathscr{A}}(z, s):=\sum_{\substack{\mathfrak{a} \in \mathscr{A} \\ \mathfrak{a}: \text { anti-int. }}} E_{\mathfrak{a} \hookrightarrow L}(z, s),
$$

where the sum is taken over the anti-integral ideals in $\mathscr{A}$, and define

$$
E_{L}(z, s):=\sum_{\mathscr{A} \in C l_{F}} E_{L, \mathscr{A}}(z, s)=\sum_{x \in \mathcal{O}_{F}^{\times} \backslash L-\{0\}} \prod_{\sigma \in S_{F}} \frac{\left|\operatorname{det} g_{\sigma}\right|^{n_{\sigma} s}}{\|\left. x g_{\sigma}\right|^{\mid n n_{\sigma} s}} .
$$

Here the last equality follows from Lemma 2.2.4 (1) and Corollary 2.2.6.

Example 2.2.8. In the case where $F=\mathbb{Q}, n=2, L=\mathbb{Z} \oplus \mathbb{Z}$, and $\mathfrak{a}=\mathbb{Z}$, we have $L_{\mathfrak{a}}=\left\{(c, d) \in \mathbb{Z}^{2} \mid(c, d)=1\right\}$, and $E_{\mathbb{Z} \hookrightarrow \mathbb{Z}^{2}}(z, s)$ is nothing but the usual real analytic Eisenstein series (1.4).

\section{Hecke's integral formula}

In this section, we first show the convergence of Eisenstein series defined in Section 2.2 . Then we prove the relative Hecke's integral formula for $E / F$. 


\subsection{Haar measures}

First, we fix the normalization of the Haar measures. Let $k$ be an arbitrary number field. For $\sigma \in S_{k}$, we normalize the Haar measure $d x_{\sigma}$ (resp. $\left.d^{\times} t_{\sigma}\right)$ on $k_{\sigma}\left(\right.$ resp. $\mathbb{R}_{>0}$ ) as

$$
\begin{aligned}
& d x_{\sigma}=d x, \quad d^{\times} t_{\sigma}=d t / t, \quad \text { if } \sigma \text { is real, } \\
& d x_{\sigma}=i d x d \bar{x}, \quad \quad d^{\times} t_{\sigma}=d t^{2} / t^{2}, \quad \text { if } \sigma \text { is complex. }
\end{aligned}
$$

Then we define the Haar measure $d x_{k}$ (resp. $d^{\times} t_{k}$ ) on $k_{\infty}$ (resp. $T_{k}$ ) to be the product measure $d x_{k}:=\prod_{\sigma \in S_{k}} d x_{\sigma}\left(\right.$ resp. $\left.d^{\times} t_{k}:=\prod_{\sigma \in S_{k}} d^{\times} t_{\sigma}\right)$.

Now, let $k^{\prime} / k$ be an arbitrary extension of number fields. We normalize the Haar measure $d^{\times} t_{k^{\prime} / k}$ on $T_{k^{\prime} / k}$ so that the induced Haar measure on the quotient group $T_{k} \simeq$ $T_{k^{\prime}} / T_{k^{\prime} / k}$ coincides with $d^{\times} t_{k}$, that is, for any integrable function $\phi$ on $T_{k^{\prime}}$, we have

$$
\int_{T_{k^{\prime}}} \phi d^{\times} t_{k^{\prime}}=\int_{T_{k}}\left(\int_{T_{k^{\prime} / k}} \phi d^{\times} t_{k^{\prime} / k}\right) d^{\times} t_{k}
$$

Next, we fix the notion of the relative gamma factor for $k^{\prime} / k$.

Lemma 3.1.1. Let $r$ be a positive integer, and let also $n_{1}, \ldots, n_{r}$ be positive integers. Set $T:=\left\{t=\left(t_{i}\right)_{i} \in \mathbb{R}_{>0}^{r} \mid \prod_{i=1}^{r} t^{n_{i}}=1\right\}$. Take any $i_{0} \in\{1, \ldots, r\}$, and let

$$
p^{i_{0}}: \mathbb{R}_{>0}^{r} \rightarrow \mathbb{R}_{>0}^{r-1}:\left(t_{i}\right)_{1 \leq i \leq r} \mapsto\left(t_{i}\right)_{1 \leq i \leq r, i \neq i_{0}}
$$

be the natural projection, which induces an isomorphism $p^{i_{0}}: T \stackrel{\sim}{\rightarrow} \mathbb{R}_{>0}^{r-1}$. We define the Haar measure $d^{\times} t$ on $T$ to be the pull-back measure $d^{\times} t:=\left(p^{i_{0}}\right)^{*} \prod_{i \neq i_{0}} d t^{n_{i}} / t^{n_{i}}$.

(1) For any integrable function $\phi$ on $\mathbb{R}_{>0}^{r}$, the measure $d^{\times} t$ satisfies

$$
\int_{\mathbb{R}_{>0}^{r}} \phi(t) \prod_{i=1}^{r} \frac{d t_{i}^{n_{i}}}{t_{i}^{n_{i}}}=\int_{u=t_{1}^{n_{1}} \ldots t_{r}^{n_{r}} \in \mathbb{R}_{>0}}\left(\int_{T} \phi(t) d^{\times} t\right) \frac{d u}{u} .
$$

In particular, the Haar measure $d^{\times} t$ is independent of the choice of $i_{0}$.

(2) Set $n:=\sum_{i=1}^{r} n_{i}$, and let $\Gamma(s)$ be the gamma function. Then

$$
n \Gamma(n s) \int_{T} \frac{1}{\left(t_{1}+\cdots+t_{r}\right)^{n s}} d^{\times} t=\prod_{i=1}^{r} n_{i} \Gamma\left(n_{i} s\right) .
$$

Proof. This can be proved by a straightforward computation, and we omit the proof.

Let $k^{\prime} / k$ be an arbitrary extension of number fields of degree $n$. We define the relative gamma factor $\Gamma_{k^{\prime} / k}(s)(s \in \mathbb{C}, \operatorname{Re}(s)>1)$ as

$$
\Gamma_{k^{\prime} / k}(s):=\int_{T_{k^{\prime} / k}} \prod_{\sigma \in S_{k}} \frac{1}{\left(\sum_{\tau \in S_{k^{\prime}, \sigma}} t_{\tau}^{2}\right)^{n n_{\sigma} s / 2}} d^{\times} t_{k^{\prime} / k} .
$$


Lemma 3.1.2. We have

$$
\Gamma_{k^{\prime} / k}(s)=\frac{\prod_{\tau \in S_{k^{\prime}}} \frac{n_{\tau}}{2} \Gamma\left(\frac{n_{\tau} s}{2}\right)}{\prod_{\sigma \in S_{k}} \frac{n n_{\sigma}}{2} \Gamma\left(\frac{n n_{\sigma} s}{2}\right)}, \quad \text { for } \operatorname{Re}(s)>1 .
$$

Proof. This follows from Lemma 3.1.1.

Let again $k$ (resp. $k^{\prime} / k$ ) be a number field (resp. an extension of number fields). We define the regulator $R_{k}$ (resp. relative regulator $R_{k^{\prime} / k}$ ) to be the volume of $T_{k / \mathbb{Q}} / U_{k}$ (resp. $T_{k^{\prime} / k} / U_{k^{\prime} / k}$ ) with respect to the Haar measure $d^{\times} t_{k / \mathbb{Q}}\left(\right.$ resp. $\left.d^{\times} t_{k^{\prime} / k}\right)$. We define $\mu_{k}$ (resp. $\mu_{k^{\prime} / k}$ ) to be the subgroup of torsion elements in $\mathcal{O}_{k}^{\times}$(resp. $\mathcal{O}_{k^{\prime} / k}^{\times}$), and set $w_{k}:=\# \mu_{k}\left(\right.$ resp. $\left.w_{k^{\prime} / k}:=\# \mu_{k^{\prime} / k}\right)$.

Lemma 3.1.3. Let $k^{\prime} / k$ be an extension of number fileds. Then we have

$$
R_{k^{\prime} / k}=\frac{\left[U_{k^{\prime}}: U_{k} U_{k^{\prime} / k}\right]}{\left[k^{\prime}: k\right]^{r_{k}-1}} \frac{R_{k^{\prime}}}{R_{k}}
$$

Proof. This follows easily from the exact sequence $1 \rightarrow U_{k^{\prime} / k} \rightarrow U_{k} U_{k^{\prime} / k} \rightarrow N_{k^{\prime} / k} U_{k} \rightarrow 1$. See also [6, Theorem 1], although their definition of the relative regulator is slightly different from ours.

\subsection{The convergence of Eisenstein series}

Now we prove the convergence of Eisenstein series defined in Section 2.2, Let $F$ be the same as in Section 2, Note that we can identify $T_{F / \mathbb{Q}}$ as a subgroup of $G(\mathbb{R})=G L_{n}\left(F_{\infty}\right)$ lying in the center $F_{\infty}^{\times} \subset G L_{n}\left(F_{\infty}\right)$.

Lemma 3.2.1. The infinite series

$$
\mathcal{E}([g], s):=\sum_{x \in L-\{0\}} \frac{1}{\left(\sum_{\sigma \in S_{F}}\left\|x g_{\sigma}\right\|^{2}\right)^{d n s / 2}}
$$

converges absolutely and compactly for $\operatorname{Re}(s)>1$ and $g=\left(g_{\sigma}\right)_{\sigma} \in G(\mathbb{R})$. Moreover, for any $g \in G(\mathbb{R})$ and $\rho \in U_{F}$, we have $\mathcal{E}_{L}([g \rho], s)=\mathcal{E}_{L}([g], s)$.

Proof. This is the well-known convergence of the Epstein zeta function. See [17, p.47] for example.

Proposition 3.2.2. We have

$$
\int_{T_{F / \mathbb{Q}} / U_{F}} \mathcal{E}([g t], s) d^{\times} t_{F / \mathbb{Q}}=\frac{w_{F} \Gamma_{F / \mathbb{Q}}(n s)}{\prod_{\sigma \in S_{F}}\left|\operatorname{det} g_{\sigma}\right|^{n_{\sigma} s}} E_{L}(z, s),
$$

for $\operatorname{Re}(s)>1$ and $g=\left(g_{\sigma}\right)_{\sigma} \in G(\mathbb{R})$. In particular, $E_{L}(z, s)$ converges absolutely and compactly for $\operatorname{Re}(s)>1$ and $g=\left(g_{\sigma}\right)_{\sigma} \in G(\mathbb{R})$. 
Proof. We see this by the classical argument as follows.

$$
\begin{aligned}
L H S & =\int_{T_{F / \mathbb{Q}} / U_{F}} \sum_{x \in L-\{0\}} \frac{1}{\left(\sum_{\sigma \in S_{F}}\left\|x g_{\sigma}\right\|^{2} t_{\sigma}^{2}\right)^{d n s / 2}} d^{\times} t_{F / \mathbb{Q}} \\
& =w_{F} \int_{T_{F / \mathbb{Q}}} \sum_{x \in \mathcal{O}_{F}^{\times} \backslash L-\{0\}} \frac{1}{\left(\sum_{\sigma \in S_{F}}\left\|x g_{\sigma}\right\|^{2} t_{\sigma}^{2}\right)^{d n s / 2}} d^{\times} t_{F / \mathbb{Q}} \\
& =w_{F} \sum_{x \in \mathcal{O}_{F}^{\times} \backslash L-\{0\}} \int_{T_{F / \mathbb{Q}}} \frac{1}{\left(\sum_{\sigma \in S_{F}}\left\|x g_{\sigma}\right\|^{2} t_{\sigma}^{2}\right)^{d n s / 2}} d^{\times} t_{F / \mathbb{Q}} .
\end{aligned}
$$

Now, since $\rho:=\left(\frac{\left\|x g_{\sigma}\right\|}{\prod_{\sigma^{\prime} \in S_{F}}\left\|x g_{\sigma^{\prime}}\right\|^{n} \sigma^{\prime} / d}\right)_{\sigma} \in T_{F / \mathbb{Q}}$, the Haar measure $d^{\times} t_{F / \mathbb{Q}}$ is invariant under the change of variables $t \mapsto \rho^{-1} t$. Then we have

$$
L H S=w_{F} \sum_{x \in \mathcal{O}_{F}^{\times} \backslash L-\{0\}} \frac{1}{\prod_{\sigma^{\prime} \in S_{F}}\left\|x g_{\sigma^{\prime}}\right\|^{n n_{\sigma^{\prime}} s}} \int_{T_{F / \mathbb{Q}}} \frac{1}{\left(\sum_{\sigma \in S_{F}} t_{\sigma}^{2}\right)^{d n s / 2}} d^{\times} t_{F / \mathbb{Q}} .
$$

The proposition now follows from (3.7).

Proposition 3.2.3. Let $\mathfrak{a} \hookrightarrow L$ be a parabolic data, and let $\mathscr{A} \in C l_{F}$.

(1) Both $E_{\mathfrak{a} \hookrightarrow L}(z, s)$ (Definition 2.2.3, Corollary 2.2.6) and $E_{L, \mathscr{A}}(z, s)$ (Definition 2.2.7) converge absolutely and compactly for $\operatorname{Re}(s)>1$ and $z \in \mathfrak{h}_{F}^{n}$.

(2) The Eisenstein series $E_{\mathfrak{a} \hookrightarrow L}(z, s)$ is an automorphic function on $\mathfrak{h}_{F}^{n}$ with respect to $\Gamma_{L}$, that is, we have

$$
E_{\mathfrak{a} \hookrightarrow L}(\gamma z, s)=E_{\mathfrak{a} \hookrightarrow L}(z, s), \quad \text { for all } \gamma \in \Gamma_{L} .
$$

(3) We have

$$
E_{L,[\mathfrak{a}]}(z, s)=\frac{\zeta_{F}\left(\mathfrak{a}^{-1}, n s\right)}{(N \mathfrak{a})^{n s}} E_{\mathfrak{a} \hookrightarrow L}(z, s) .
$$

Proof. (1) follows directly from Proposition 3.2.2, and (2) follows directly from the definition of $E_{\mathfrak{a} \hookrightarrow L}(z, s)$ (Definition 2.2.3). To prove (3), it suffices to see

$$
E_{\alpha \mathfrak{a} \hookrightarrow L}(z, s)=\left|N_{F / \mathbb{Q}}(\alpha)\right|^{n s} E_{\mathfrak{a} \hookrightarrow L}(z, s), \quad \text { for } \alpha \in F^{\times} .
$$

This follows from the identity $L_{\alpha \mathfrak{a}}=\frac{1}{\alpha} L_{\mathfrak{a}}$ and Corollary 2.2.6, 


\subsection{Relative Hecke's integral formula for $E / F$}

Now we prove our first main theorem. Let the notations be the same as in Section 2.1. That is, $E / F$ is an extension of number fields of degree $n, \varpi$ is the Heegner object associated to a basis $w$ of $E$ over $F, L \subset F^{n}$ is the $\mathcal{O}_{F}$-lattice corresponding to a fractional $\mathcal{O}_{E}$-ideal $\mathfrak{A}$ with respect to the basis $w$, and $\Gamma=\Gamma_{L}$ is the arithmetic subgroup of $S L_{n}(F)$ associated to $L$. Let $\mathfrak{a}$ be an anti-integral $\mathcal{O}_{F}$-ideal and let $\mathfrak{a} \hookrightarrow L$ be a parabolic data. We consider the Eisenstein series $E_{\mathfrak{a} \hookrightarrow L}(z, s)$ associated to this data. In this section, we assume $\operatorname{Re}(s)>1$.

Theorem 3.3.1. Put $\Delta_{w}:=N_{F / \mathbb{Q}}\left(d_{w}\right)$, where $d_{w}:=(\operatorname{det} W)^{2} \in F$ is the "discriminant" of the basis $w$. Then we have

$$
\begin{aligned}
& \int_{T_{E / F} / U_{E / F}} E_{\mathfrak{a} \hookrightarrow L}(\bar{\varpi}(t), s) d^{\times} t_{E / F} \\
& =\left|\Delta_{w}\right|^{\frac{s}{2}} R_{E / F} \frac{w_{E} R_{E}^{-1} \Gamma_{E / \mathbb{Q}}(s)}{w_{F} R_{F}^{-1} \Gamma_{F / \mathbb{Q}}(n s)} \sum_{x \in \mathcal{O}_{E}^{\times} \backslash \mathfrak{A}_{\mathfrak{a}}} \frac{1}{\left|N_{E / \mathbb{Q}}(x)\right|^{s}},
\end{aligned}
$$

for $\operatorname{Re}(s)>1$, where $\mathfrak{A}_{\mathfrak{a}}$ is the image of $L_{\mathfrak{a}}$ under the isomorphism $w$ 2.7), or equivalently, the subset of $\mathfrak{A}$ defined in the same way as (2.22).

Proof. The proof is similar to that of Proposition 3.2.2, Let $W=\left(W_{\sigma}\right)_{\sigma} \in G(\mathbb{R})$, and $I(t)=\left(I(t)_{\sigma}\right)_{\sigma} \in G(\mathbb{R})\left(t \in T_{E / F}\right)$ be as in Section 2.1 so that $\varpi(t)=[W I(t)]$. Let us fix a maximal torsion free subgroup $\tilde{U}_{E}$ (resp. $\left.\tilde{U}_{F}, \tilde{U}_{E / F}\right)$ of $\mathcal{O}_{E}^{\times}\left(\operatorname{resp} . \mathcal{O}_{F}^{\times}, \mathcal{O}_{E / F}^{\times}\right)$so that $\tilde{U}_{F}, \tilde{U}_{E / F} \subset \tilde{U}_{E}$. Note that we have $\operatorname{reg}_{E / F}^{\times}: \tilde{U}_{E / F} \stackrel{\sim}{\rightarrow} U_{E / F}$, etc. Using Corollary 2.2.6, we obtain

$$
\begin{aligned}
L H S & =\frac{1}{w_{F}} \int_{T_{E / F} / U_{E / F}} \sum_{x \in \tilde{U}_{F} \backslash L_{\mathfrak{a}}} \prod_{\sigma} \frac{\left|\operatorname{det} W_{\sigma}\right|^{n_{\sigma} s}}{\left\|x W_{\sigma} I(t)_{\sigma}\right\|^{n n_{\sigma} s}} d^{\times} t_{E / F} \\
& =\frac{1}{w_{F}} \int_{T_{E / F} / U_{E / F}} \sum_{u \in \tilde{U}_{E / F}} \sum_{x \in \tilde{U}_{F} \tilde{U}_{E / F} \backslash L_{\mathfrak{a}}} \prod_{\sigma} \frac{\left|\operatorname{det} W_{\sigma}\right|^{n_{\sigma} s}}{\left\|x \varrho_{w}(u) W_{\sigma} I(t)_{\sigma}\right\|^{n n_{\sigma} s}} d^{\times} t_{E / F} \\
& =\frac{1}{w_{F}} \int_{T_{E / F} / U_{E / F}} \sum_{u \in U_{E / F}} \sum_{x \in \tilde{U}_{F} \tilde{U}_{E / F} \backslash L_{\mathfrak{a}}} \prod_{\sigma} \frac{\left|\operatorname{det} W_{\sigma}\right|^{n_{\sigma} s}}{\left\|x W_{\sigma} I(u t)_{\sigma}\right\|^{n n_{\sigma} s}} d^{\times} t_{E / F} \\
& =\frac{1}{w_{F}} \int_{T_{E / F}} \sum_{x \in \tilde{U}_{F} \tilde{U}_{E / F} \backslash L_{\mathfrak{a}}} \prod_{\sigma} \frac{\left|\operatorname{det} W_{\sigma}\right|^{n_{\sigma} s}}{\left\|x W_{\sigma} I(t)_{\sigma}\right\|^{n n_{\sigma} s}} d^{\times} t_{E / F}
\end{aligned}
$$

Here, the action of $\tilde{U}_{E / F}$ on $L_{\mathfrak{a}}$ is defined by the regular representation $\varrho_{w}$, and the equality (3.22) follows from Lemma 2.1.3. By putting $z=x w$, we obtain

$$
L H S=\frac{\left|\Delta_{w}\right|^{\frac{s}{2}}}{w_{F}} \sum_{z \in \tilde{U}_{F} \tilde{U}_{E / F} \backslash \mathfrak{A}_{\mathfrak{a}}} \int_{T_{E / F}} \prod_{\sigma} \frac{1}{\left(\sum_{\tau \in S_{E, \sigma}}|\tau(z)|^{2} t_{\tau}^{2}\right)^{n n_{\sigma} s / 2}} d^{\times} t_{E / F}
$$


Now, since $\rho:=\left(|\tau(z)| \prod_{\tau^{\prime} \in S_{E, \sigma_{\tau}}}\left|\tau^{\prime}(z)\right|^{-n_{\tau^{\prime}} / n n_{\sigma_{\tau}}}\right)_{\tau} \in T_{E / F}$, where $\sigma_{\tau}$ is the place of $F$ below $\tau$, the Haar measure $d^{\times} t_{E / F}$ is invariant under the change of variables $t \mapsto \rho^{-1} t$. Therefore, we have

$$
\begin{aligned}
L H S & =\frac{\left|\Delta_{w}\right|^{\frac{s}{2}}}{w_{F}} \sum_{z \in \tilde{U}_{F} \tilde{U}_{E / F} \backslash \mathfrak{A}_{\mathfrak{a}}} \frac{1}{\left|N_{E / \mathbb{Q}}(z)\right|^{s}} \int_{T_{E / F}} \prod_{\sigma} \frac{1}{\left(\sum_{\tau \in S_{E, \sigma}} t_{\tau}^{2}\right)^{n n_{\sigma} s / 2}} d^{\times} t_{E / F} \\
& =\left|\Delta_{w}\right|^{\frac{s}{2}}\left[U_{E}: U_{F} U_{E / F}\right] \Gamma_{E / F}(s) \frac{w_{E}}{w_{F}} \sum_{z \in \mathcal{O}_{E}^{\times} \backslash \mathfrak{A}_{\mathfrak{a}}} \frac{1}{\left|N_{E / \mathbb{Q}}(z)\right|^{s}}
\end{aligned}
$$

Now the theorem follows from Lemma 3.1.2 and Lemma 3.1.3.

Definition 3.3.2. Let $\mathfrak{A}$ be the same as above. For $\mathscr{A} \in C l_{F}$, we define the partial zeta function $\zeta_{E / F, \mathscr{A}}\left(\mathfrak{A}^{-1}, s\right)$ associated to $\mathfrak{A}^{-1}$ relative to $\mathscr{A}$ as

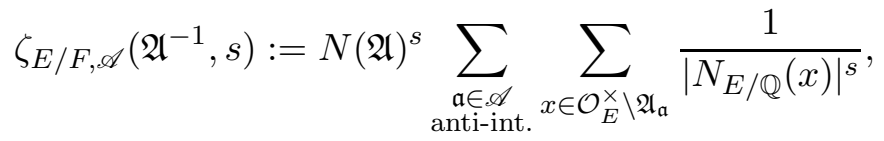

where $N(\mathfrak{A})$ is the absolute norm of the fractional $\mathcal{O}_{E}$-ideal $\mathfrak{A}$.

Now, using Proposition 3.2 .3 (3), we can rewrite Theorem 3.3.1 as follows.

Theorem 3.3.3 (Relative Hecke's integral formula).

Let $[\mathfrak{a}] \in C l_{F}$ be the ideal class of $\mathfrak{a}$. Put

$$
c_{E / F}(s):=R_{E / F} \frac{w_{E} R_{E}^{-1} \Gamma_{E / \mathbb{Q}}(s)}{w_{F} R_{F}^{-1} \Gamma_{F / \mathbb{Q}}(n s)} .
$$

Then we obtain

$$
\int_{T_{E / F} / U_{E / F}} E_{\mathfrak{a} \hookrightarrow L}(\bar{\varpi}(t), s) d^{\times} t_{E / F}=\left|\Delta_{w}\right|^{\frac{s}{2}} c_{E / F}(s) \frac{N(\mathfrak{A})^{-s}}{N(\mathfrak{a})^{-n s}} \frac{\zeta_{E / F,[\mathfrak{a}]}\left(\mathfrak{A}^{-1}, s\right)}{\zeta_{F}\left(\mathfrak{a}^{-1}, n s\right)} .
$$

Corollary 3.3.4. Taking the sum over the anti-integral ideals $\mathfrak{a}$, we obtain

$$
\int_{T_{E / F} / U_{E / F}} E_{L}(\bar{\varpi}(t), s) d^{\times} t_{E / F}=\left|\Delta_{w}\right|^{\frac{s}{2}} c_{E / F}(s) N(\mathfrak{A})^{-s} \zeta_{E}\left(\mathfrak{A}^{-1}, s\right) .
$$

Remark. In the case where $F=\mathbb{Q}$ or $n=2$, Corollary 3.3.4 gives the results of Hecke [10, p. 370] and Yamamoto [18, Theorem 3.1.2] respectively. It should be also remarked that in the case where $F$ is imaginary quadratic and $n=2$, or $F=\mathbb{Q}$ and $E$ is totally real, Harder [9] and Sczech [16] respectively obtain results which can be seen as a "cohomological interpretation" of this theorem, and deduce the rationality of the special values of zeta functions. 


\section{The Fourier expansion of Eisenstein series}

In this preliminary section, we present a kind of the Fourier expansion formula of the Eisenstein series $E_{L,[\mathfrak{a}]}(z, s)$ for $\operatorname{Re}(s)>1$. This shows the analytic continuation of the Eisenstein series, and enables us to compute its residue and the constant term at $s=1$. As remarked in Section 1, the Fourier coefficients (Theorem 4.2.1) may be obtained from the more general theory. However here we present an explicit formula in terms of ideal classes of $F$, and give a proof in a self-contained way.

\subsection{Setting}

Let $F / \mathbb{Q}$ be a number field of degree $d$, and let $\mathfrak{a} \hookrightarrow L$ be as before. For another $\mathcal{O}_{F^{-}}$ lattice $L^{\prime} \subset F^{n}$ which is isomorphic to $L$, let $\gamma \in G L_{n}(F)$ be a matrix such that $L \gamma=L^{\prime}$. Let us consider the parabolic data $\mathfrak{a} \hookrightarrow L \stackrel{\gamma}{\rightarrow} L^{\prime}$. Then we easily see that

$$
E_{\mathfrak{a} \hookrightarrow L}(z, s)=\left|N_{F / \mathbb{Q}}(\operatorname{det} \gamma)\right|^{s} E_{\mathfrak{a} \hookrightarrow L^{\prime}}\left(\gamma^{-1} z, s\right) .
$$

On the other hand, by Proposition 2.2.5 any $\mathcal{O}_{F}$-lattice $L \subset F^{n}$ is isomorphic to the lattice of the form $\mathfrak{a}_{1} \oplus \cdots \oplus \mathfrak{a}_{n} \subset F^{n}$ for some anti-integral ideals $\mathfrak{a}_{i} \subset F(i=1, \ldots, n)$. Therefore, in this section we assume $L=\mathfrak{a}_{1} \oplus \cdots \oplus \mathfrak{a}_{n} \subset F^{n}$. We also assume $n \geq 2$.

Let $\mathfrak{a}_{n} \stackrel{i_{n}}{\longrightarrow} L$ be the $n$-th inclusion, which is another parabolic data. We consider the Fourier expansion of the Eisenstein series $E_{L,[\mathfrak{a}]}(z, s)$ at the "cusp" corresponding to the parabolic subgroup $P_{\mathfrak{a}_{n} \hookrightarrow L}=\operatorname{Stab}_{\Gamma_{L}}\left(\mathfrak{a}_{n} \hookrightarrow L\right)$ associated to the data $\mathfrak{a}_{n} \hookrightarrow L$. Let

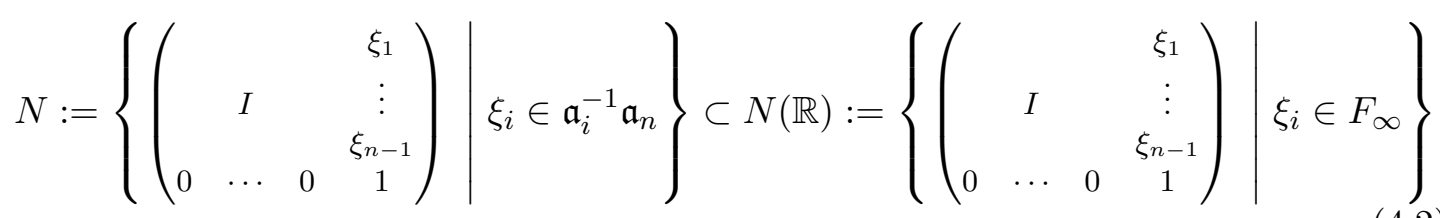

be the nilpotent radical of $P_{\mathfrak{a}_{n} \hookrightarrow L}$, and its canonical lifting into $G(\mathbb{R})$. In the following, we identify $N(\mathbb{R})$ with $F_{\infty}^{n-1}$ via the isomorphism

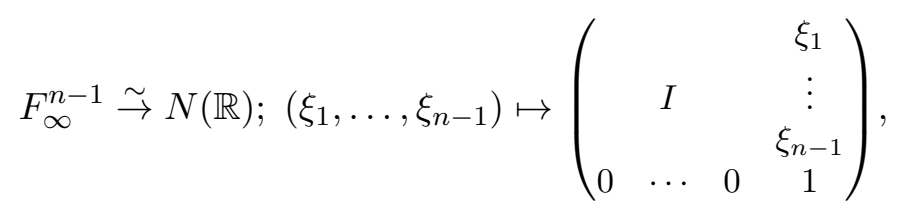

which clearly induces an isomorphism $\mathfrak{a}_{1}^{-1} \mathfrak{a}_{n} \oplus \cdots \oplus \mathfrak{a}_{n-1}^{-1} \mathfrak{a}_{n} \stackrel{\sim}{\rightarrow} N$. We also identify $N$ with the $\mathcal{O}_{F}$-lattice $\mathfrak{a}_{1}^{-1} \mathfrak{a}_{n} \oplus \cdots \oplus \mathfrak{a}_{n-1}^{-1} \mathfrak{a}_{n}$ in $F_{\infty}^{n-1}$ via this isomorphism. We denote by $d \xi$ the Haar measure on $N(\mathbb{R}) \simeq F_{\infty}^{n-1}$ which is the product measure of $d x_{F}$ on $F_{\infty}$ defined in Section 3.1. We denote by

$$
N^{\vee}:=\left\{y \in F_{\infty}^{n-1} \mid\langle x, y\rangle \in \mathbb{Z}, \forall x \in N\right\}=\mathfrak{a}_{1} \mathfrak{a}_{n}^{-1} \mathfrak{d}_{F}^{-1} \oplus \cdots \oplus \mathfrak{a}_{n-1} \mathfrak{a}_{n}^{-1} \mathfrak{d}_{F}^{-1}
$$

the dual lattice of $N$ with respect to the pairing $\langle\rangle:, F_{\infty}^{n-1} \times F_{\infty}^{n-1} \rightarrow \mathbb{R}$. 
Definition 4.1.1 (Fourier coefficients). For $* \in\{\mathfrak{a} \hookrightarrow L,(L,[\mathfrak{a}])\}$ and $\nu \in N^{\vee}$, we define the $\nu$-th Fourier coefficient of $E_{*}(z, s)$ as

$$
\mathscr{I}_{*, \nu}(z, s):=\frac{1}{\operatorname{vol}(N \backslash N(\mathbb{R}))} \int_{N \backslash N(\mathbb{R})} E_{*}(\xi z, s) e^{-2 \pi i\langle\nu, \xi\rangle} d \xi .
$$

Then, by the general theory of the Fourier expansion, we have

$$
E_{*}(z, s)=\sum_{\nu \in N^{\vee}} \mathscr{I}_{*, \nu}(z, s)
$$

Iwasawa normal form In order to state the Fourier expansion formula explicitly, for $z \in \mathfrak{h}_{F}^{n}$, we always take a representative $g \in G(\mathbb{R})=G L_{n}\left(F_{\infty}\right)$ of $z$ (i.e., $z=[g]$ ) of the following form called the Iwasawa normal form: $g=X Y$ with

$$
X=\left(\begin{array}{ccc}
1 & & x_{i j} \\
& \ddots & \\
0 & & 1
\end{array}\right), \quad Y=\left(\begin{array}{cccc}
y_{1}^{\prime} & & & 0 \\
& \ddots & & \\
0 & & y_{n-1}^{\prime} & \\
0 & & & 1
\end{array}\right)
$$

where $x_{i j} \in F_{\infty}$, and $y_{i}^{\prime}=y_{i} \cdots y_{n-1}$ for $y_{i} \in T_{F}=\prod_{\sigma} \mathbb{R}_{>0}$. Note that the existence and the uniqueness of the Iwasawa normal form is guaranteed by the Iwasawa decomposition of $G L_{n}(\mathbb{R})$ and $G L_{n}(\mathbb{C})$. With the above notation, for $2 \leq j \leq n$, we set $\mathbf{x}_{j}:={ }^{t}\left(x_{1 j}, \ldots, x_{j-1, j}\right) \in F_{\infty}^{j-1}$, which is essentially the $j$-th column of $X$. Furthermore, for $1 \leq j \leq n-1$, we put

$$
X^{(j)}:=\left(\begin{array}{cccc}
1 & x_{12} & \cdots & x_{1, n-j} \\
& \ddots & \ddots & \vdots \\
& & \ddots & x_{n-j-1, n-j} \\
0 & & & 1
\end{array}\right), \quad Y^{(j)}:=\left(\begin{array}{cccc}
y_{1} \cdots y_{n-j} & & & 0 \\
& y_{2} \cdots y_{n-j} & & \\
& & \ddots & \\
0 & & & y_{n-j}
\end{array}\right),
$$

and set $g^{(j)}:=X^{(j)} Y^{(j)} \in G L_{n-j}\left(F_{\infty}\right)$. We fix these notations throughout the paper.

Some arithmetic functions and Bessel function We prepare some arithmetic functions and Bessel function.

Let $\mathfrak{m} \subset \mathcal{O}_{F}$ be a non-zero integral ideal. We define

- (Euler's totient function) $\varphi(\mathfrak{m}):=\#\left(\mathcal{O}_{F} / \mathfrak{m}\right)^{\times}$,

- (Divisor sum) $\sigma_{s}(\mathfrak{m}, \chi):=\sum_{\mathfrak{n} \mid \mathfrak{m}} \chi(\mathfrak{n}) N \mathfrak{n}^{s}, \quad\left(\chi \in \operatorname{Hom}\left(C l_{F}, \mathbb{C}^{\times}\right), s \in \mathbb{C}\right)$.

Furthermore, let $\mathfrak{b} \subset F$ be a fractional $\mathcal{O}_{F}$-ideal such that $\mathfrak{b} \subset \mathfrak{d}_{F}^{-1}$. Then, since $\mathcal{O}_{F}$ is a Dedekind domain, $\mathcal{O}_{F} / \mathfrak{m}$ is a principal ideal ring, and thus $\mathfrak{b m}^{-1} / \mathfrak{b}$ is isomorphic to $\mathcal{O}_{F} / \mathfrak{m}$ as an $\mathcal{O}_{F} / \mathfrak{m}$-module. We denote by $\left(\mathfrak{b m}^{-1} / \mathfrak{b}\right)^{\times}$the set of generators of $\mathfrak{b m}^{-1 / \mathfrak{b}}$ as an $\mathcal{O}_{F} / \mathfrak{m}$-module. We define the special case of the Gauss sum as 
- (Ramanujan sum) $\tau(\mathfrak{m}, \mathfrak{b}):=\sum_{x \in\left(\mathfrak{b} \mathfrak{m}^{-1} / \mathfrak{b}\right)^{\times}} e^{2 \pi i T r_{F / \mathbb{Q}}(x)}$,

where the sum is taken over a system of representatives of $\left(\mathfrak{b m}^{-1 / \mathfrak{b})^{\times}}\right.$. This is welldefined since $\mathfrak{b} \subset \mathfrak{d}_{F}^{-1}$.

Lemma 4.1.2. Let $\mathfrak{m} \subset \mathcal{O}_{F}$ be a non-zero integral ideal, and let $\mathfrak{b} \subset F$ be a fractional $\mathcal{O}_{F}$-ideal such that $\mathfrak{b} \subset \mathfrak{d}_{F}^{-1}$, then we have

$$
\sum_{\mathfrak{a} \mid \mathfrak{m}} \varphi(\mathfrak{a})=N \mathfrak{m}, \quad \sum_{\mathfrak{a} \mid \mathfrak{m}} \tau(\mathfrak{a}, \mathfrak{b})= \begin{cases}N \mathfrak{m} & \left(\mathfrak{m} \mid \mathfrak{b} \mathfrak{d}_{F}\right) \\ 0 & (\text { otherwise })\end{cases}
$$

Proof. This follows directly from the identity $\mathfrak{b m}^{-1} / \mathfrak{b}=\coprod_{\mathfrak{a} \mid \mathfrak{m}}\left(\mathfrak{b} \mathfrak{a}^{-1} / \mathfrak{b}\right)^{\times}$.

Definition 4.1.3. Let $\mathbf{s}=\left(s_{1}, \ldots, s_{k}\right) \in \mathbb{C}^{k}, \mathbf{t}=\left(t_{1}, \ldots, t_{l}\right) \in \mathbb{C}^{l}$ be tuples of complex variables such that $\operatorname{Re}\left(s_{i}\right), \operatorname{Re}\left(t_{j}\right)>2$ for all $i, j$, and let $\mathfrak{b}_{j} \subset \mathfrak{d}_{F}^{-1} 1 \leq j \leq l$ be fractional $\mathcal{O}_{F}$-ideals. For $\mathscr{A} \in C l_{F}$, we define

$$
Z_{\mathscr{A}}\left(\mathbf{s} ; \mathbf{t} ;\left(\mathfrak{b}_{j}\right)_{j}\right):=\sum_{\substack{\mathfrak{m}_{1}, \ldots, \mathfrak{m}_{k} \subset \mathcal{O}_{F} \\ \mathfrak{n}_{1}, \ldots, \mathfrak{n}_{C} \subset \mathcal{O}_{F} \\ \mathfrak{m}_{1} \cdots \mathfrak{m}_{k} \mathfrak{n}_{1} \cdots \mathfrak{n}_{l} \in \mathscr{A}}} \prod_{i=1}^{k} \frac{\varphi\left(\mathfrak{m}_{i}\right)}{N \mathfrak{m}_{i}^{s_{i}}} \prod_{j=1}^{l} \frac{\tau\left(\mathfrak{n}_{j}, \mathfrak{b}_{j}\right)}{N \mathfrak{n}_{j}^{t_{j}}}
$$

if $k, l \geq 1$. In the case where $k=0$ or $l=0$, we define

$$
\begin{aligned}
& Z_{\mathscr{A}}(\emptyset, \emptyset):=\left\{\begin{array}{ll}
1 & \left(\mathscr{A}=\left[\mathcal{O}_{F}\right]\right) \\
0 & \left(\mathscr{A} \neq\left[\mathcal{O}_{F}\right]\right)
\end{array}, \quad Z_{\mathscr{A}}(\mathbf{s} ; \emptyset):=\sum_{\substack{\mathfrak{m}_{1}, \ldots, \mathfrak{m}_{k} \subset \mathcal{O}_{F} \\
\mathfrak{m}_{1} \cdots \mathfrak{m}_{k} \in \mathscr{A}}} \prod_{i=1}^{k} \frac{\varphi\left(\mathfrak{m}_{i}\right)}{N \mathfrak{m}_{i}^{s_{i}}},\right. \\
& Z_{\mathscr{A}}\left(\emptyset ; \mathbf{t} ;\left(\mathfrak{b}_{j}\right)_{j}\right):=\sum_{\substack{\mathfrak{n}_{1}, \ldots, \mathfrak{n}_{l} \subset \mathcal{O}_{F} \\
\mathfrak{n}_{1} \cdots \mathfrak{n}_{l} \in \mathscr{A}}} \prod_{j=1}^{l} \frac{\tau\left(\mathfrak{n}_{j}, \mathfrak{b}_{j}\right)}{N \mathfrak{n}_{j}^{t_{j}}} .
\end{aligned}
$$

Furthermore, for a character $\chi \in \operatorname{Hom}\left(C l_{F}, \mathbb{C}^{\times}\right)$, we define

$$
Z\left(\mathbf{s} ; \mathbf{t} ;\left(\mathfrak{b}_{j}\right)_{j} ; \chi\right):=\sum_{\mathscr{A} \in C l_{F}} \chi(\mathscr{A}) Z_{\mathscr{A}}\left(\mathbf{s} ; \mathbf{t} ;\left(\mathfrak{b}_{j}\right)_{j}\right)
$$

Lemma 4.1.4. Let the notations be as above. Then $Z_{\mathscr{A}}\left(\mathbf{s} ; \mathbf{t} ;\left(\mathfrak{b}_{j}\right)_{j}\right)$ converges absolutely and compactly for $\operatorname{Re}\left(s_{i}\right), \operatorname{Re}\left(t_{j}\right)>2$. Moreover, for $\chi \in \operatorname{Hom}\left(C l_{F}, \mathbb{C}^{\times}\right)$, we have

$$
Z\left(\mathbf{s} ; \mathbf{t} ;\left(\mathfrak{b}_{j}\right)_{j} ; \chi\right)=\prod_{i=1}^{k} \frac{L\left(s_{i}-1, \chi\right)}{L\left(s_{i}, \chi\right)} \prod_{j=1}^{l} \frac{\sigma_{1-t_{j}}\left(\mathfrak{b}_{j} \mathfrak{d}_{F}, \chi\right)}{L\left(t_{j}, \chi\right)},
$$

where $L(s, \chi)$ is the Hecke $L$-function associated to the character $\chi$. 
Proof. This follows from Lemma 4.1.2,

On the other hand, we have

$$
Z_{\mathscr{A}}\left(\mathbf{s} ; \mathbf{t} ;\left(\mathfrak{b}_{j}\right)_{j}\right)=\frac{1}{h_{F}} \sum_{\chi \in \operatorname{Hom}\left(C l_{F}, \mathbb{C} \times\right)} \chi\left(\mathscr{A}^{-1}\right) Z\left(\mathbf{s} ; \mathbf{t} ;\left(\mathfrak{b}_{j}\right)_{j} ; \chi\right) .
$$

Therefore we get the following:

Corollary 4.1.5. For $\mathscr{A} \in C l_{F}$, we have

$$
Z_{\mathscr{A}}\left(\mathbf{s} ; \mathbf{t} ;\left(\mathfrak{b}_{j}\right)_{j}\right)=\frac{1}{h_{F}} \sum_{\chi \in \operatorname{Hom}\left(C l_{F}, \mathbb{C}^{\times}\right)} \chi\left(\mathscr{A}^{-1}\right) \prod_{i=1}^{k} \frac{L\left(s_{i}-1, \chi\right)}{L\left(s_{i}, \chi\right)} \prod_{j=1}^{l} \frac{\sigma_{1-t_{j}}\left(\mathfrak{b}_{j} \mathfrak{d}_{F}, \chi\right)}{L\left(t_{j}, \chi\right)} .
$$

In particular $Z_{\mathscr{A}}\left(\mathbf{s} ; \mathbf{t} ;\left(\mathfrak{b}_{j}\right)_{j}\right)$ can be continued meromorphically to whole $s_{i}, t_{j} \in \mathbb{C}$.

In this section, we use a slightly different gamma factor from those in Section 3, For a number field $k / \mathbb{Q}$ of degree $d$, define

$$
\Gamma_{k}(s):=\prod_{\sigma \in S_{k}} \Gamma\left(\frac{n_{\sigma} s}{2}\right) .
$$

Let $K_{s}(x)\left(s \in \mathbb{C}, x \in \mathbb{R}_{>0}\right)$ be the $K$-Bessel function, that is,

$$
K_{s}(x)=\frac{1}{2} \int_{0}^{\infty} e^{-\frac{1}{2} x\left(u+\frac{1}{u}\right)} u^{s} \frac{d u}{u} .
$$

Then, for $s \in \mathbb{C}$ and $x=\left(x_{\sigma}\right)_{\sigma} \in T_{k}$, we define the $K$-Bessel function $K_{k}(s, x)$ over $k$ as

$$
K_{k}(s, x):=\prod_{\sigma \in S_{k}} K_{\frac{n_{\sigma} s}{2}}\left(x_{\sigma}\right) .
$$

\subsection{Statement of the Fourier expansion formula}

We have the following formulas for the Fourier coefficients $\mathscr{I}_{L,[a], \nu}(z, s)$. For $1 \leq j \leq$ $n-1$, we set $L^{(j)}:=\mathfrak{a}_{1} \oplus \cdots \oplus \mathfrak{a}_{n-j} \subset F^{n-j}$.

Theorem 4.2.1. (1) (Constant term, $\nu=0$ ) For $\operatorname{Re}(s)>1$, we have

$$
\begin{aligned}
& \frac{\mathscr{I}_{L,[\mathfrak{a}], 0}(z, s)}{\zeta_{F}\left(\mathfrak{a}^{-1}, n s\right)}=\delta_{[\mathfrak{a}],\left[\mathfrak{a}_{n}\right]}\left(N \mathfrak{a}_{n}\right)^{-n s} \prod_{\sigma}\left|\operatorname{det} g_{\sigma}^{(1)}\right|^{n_{\sigma} s} \\
& +\frac{2^{r_{2}(F)} \pi^{\frac{d}{2}}}{\sqrt{\left|d_{F}\right|} N \mathfrak{a}_{n}} \frac{\Gamma_{F}(n s-1)}{\Gamma_{F}(n s)} \prod_{\sigma}\left|\operatorname{det} g_{\sigma}^{(1)}\right|^{n_{\sigma} \frac{1-s}{n-1}} \sum_{\substack{\mathfrak{m} \subset \mathcal{O}_{F} \\
\mathfrak{m} \neq 0}} \frac{\varphi(\mathfrak{m})}{N \mathfrak{m}^{n s}} \frac{E_{L^{(1)},[\mathfrak{a m}-1]}\left(\left[g^{(1)}\right], \frac{n s-1}{n-1}\right)}{\zeta_{F}\left(\mathfrak{a}^{-1} \mathfrak{m}, n s-1\right)}
\end{aligned}
$$

where $\delta$ is the Kronecker delta, $g_{\sigma}^{(1)}$ is the $\sigma$-component of $g^{(1)}$ and we identify $\left[g^{(1)}\right] \in \mathfrak{h}_{F}^{n-1}$, and $E_{L^{(1)},\left[\mathfrak{a m}^{-1}\right]}\left(\left[g^{(1)}\right], s\right)$ is the Eisenstein series on $\mathfrak{h}_{F}^{n-1}$. 
(2) (Non-constant terms, $\nu \neq 0$ ) For $\operatorname{Re}(s)>1$, we have

$$
\begin{gathered}
\sum_{\nu \in N^{\vee}-\{0\}} \frac{\mathscr{I}_{L,[a], \nu}(z, s)}{\zeta_{F}\left(\mathfrak{a}^{-1}, n s\right)}=\frac{2^{d} \pi^{\frac{d n s}{2}}}{\sqrt{\left|d_{F}\right|}\left(N \mathfrak{a}_{n}\right)^{n s}} \sum_{\substack{\mathfrak{n} \subset \mathcal{O}_{F} \\
\mathfrak{n} \neq 0}} N\left(\mathfrak{n} \mathfrak{d}_{F}^{-1}\right)^{n s-1} \sum_{\mathfrak{m} \in\left[\mathfrak{a} \mathfrak{a}_{n}^{-1} \mathfrak{n} \mathfrak{d}_{F}^{-1}\right]} \frac{\tau\left(\mathfrak{m}, \mathfrak{n} \mathfrak{d}_{F}^{-1}\right)}{N \mathfrak{m}^{n s}} \\
\times \sum_{\nu \in\left(N^{\vee}\right)_{\mathfrak{n}}-1} e^{2 \pi i\left\langle\nu, \mathbf{x}_{n}\right\rangle} \frac{K_{F}\left(n s-1,\left(2 \pi\left\|\nu g_{\sigma}^{(1)}\right\|\right)_{\sigma}\right)}{\Gamma_{F}(n s)} \prod_{\sigma} \frac{\left|\operatorname{det} g_{\sigma}^{(1)}\right|^{n_{\sigma} s}}{\left\|\nu g_{\sigma}^{(1)}\right\|^{\frac{n_{\sigma}}{2}(n s-1)}} .
\end{gathered}
$$

We give a proof of this theorem in Section 4.3.

Remark. We can deduce the corresponding results of Epstein [7, Liu-Masri [14] and Yamamoto [18] from this theorem. We go further to get rid of the Eisenstein series in the constant term. Note that in order to deduce the result in [7] and [14, we use the functional equation of the Eisenstein series, and specialize this formula to $s=0$ (see also Theorem 4.2 .3 below).

Fourier expansion formula By using Theorem 4.2.1 recursively, we obtain a kind of Fourier expansion formula for $E_{L,[\mathfrak{a}]}(z, s)$. In order to simplify the presentation, we first define some additional notation. For $0 \leq j \leq n-1$, we set

$$
\begin{aligned}
& c_{j}(z, s):=\left(\frac{2^{r_{2}(F)} \pi^{\frac{d}{2}}}{\sqrt{\left|d_{F}\right|}}\right)^{j} \frac{\Gamma_{F}(n s-j)}{\Gamma_{F}(n s)} \frac{\left(N \mathfrak{a}_{i}\right)^{j-n s}}{N \mathfrak{a}_{n} \cdots N \mathfrak{a}_{n-j+1}} \\
& \quad \times \prod_{k=1}^{n-j-1}\left|N_{F / \mathbb{Q}}\left(y_{k}\right)\right|^{k s} \prod_{k=n-j}^{n-1}\left|N_{F / \mathbb{Q}}\left(y_{k}\right)\right|^{(n-k)(1-s)}, \\
& d_{j}(z, s):=\frac{2^{d} \pi^{\frac{d s}{2}(n-j)}}{\sqrt{\left|d_{F}\right|} \Gamma_{F}(n s-j)} c_{j}(z, s)
\end{aligned}
$$

where we define $c_{0}(z, s):=\prod_{k=1}^{n-1}\left|N_{F / \mathbb{Q}}\left(y_{k}\right)\right|^{k s}$ for $j=0$. For $1 \leq j \leq n-1$, set $\Lambda^{(j)}:=\mathfrak{a}_{1} \mathfrak{a}_{n-j+1}^{-1} \mathfrak{d}_{F}^{-1} \oplus \cdots \oplus \mathfrak{a}_{n-j} \mathfrak{a}_{n-j+1}^{-1} \mathfrak{d}_{F}^{-1}$. Note that $\Lambda^{(1)}=N^{\vee}$.

Definition 4.2.2. (1) For $0 \leq j \leq n-1, \operatorname{Re}(s)>1$, we define

$$
\Phi_{j}(z, s):=c_{j}(z, s) Z_{\left[\mathfrak{a a}_{n-j}^{-1}\right]}(\overbrace{n s, n s-1, \ldots, n s-j+1}^{j-\text { tuple }} ; \emptyset),
$$

where we assume $(n s, n s-1 \ldots, n s-j+1)$ is the empty tuple $\emptyset$ if $j=0$. 
(2) For $0 \leq j \leq n-2, \operatorname{Re}(s)>1$, we define

$$
\begin{aligned}
& \Psi_{j}(z, s):=d_{j}(z, s) \sum_{\substack{\mathfrak{n} \subset \mathcal{O}_{F} \\
\mathfrak{n} \neq 0}} Z_{\left[\mathfrak{a a}_{n-j}^{-1} \mathfrak{n} \mathfrak{d}_{F}^{-1}\right]}(\overbrace{n s, n s-1, \ldots, n s-j+1}^{j-\text { tuple }} ; n s-j ; \mathfrak{n} \mathfrak{d}_{F}^{-1}) \\
& \times N\left(\mathfrak{n} \mathfrak{d}_{F}^{-1}\right)^{n s-j-1} \sum_{\nu \in\left(\Lambda^{(j+1)}\right)_{\mathfrak{n}-1}} e^{2 \pi i\left\langle\nu, \mathbf{x}_{n-j}\right\rangle} \frac{K_{F}\left(n s-j-1,\left(2 \pi\left\|\nu g_{\sigma}^{(j+1)}\right\|\right)_{\sigma}\right)}{\prod_{\sigma}\left\|\nu g_{\sigma}^{(j+1)}\right\| \|^{\frac{n_{\sigma}}{2}(n s-j-1)}}
\end{aligned}
$$

where we assume $(n s, n s-1 \ldots, n s-j+1)=\emptyset$ if $j=0$, as above.

Theorem 4.2.3. The functions $\Phi_{j}(z, s)$ and $\Psi_{j}(z, s)$ can be continued meromorphically to whole $s \in \mathbb{C}$. Furthermore, $\Phi_{j}(z, s)$ (resp. $\Psi_{j}(z, s)$ ) is holomorphic outside the poles of $\frac{L(n s-j, \chi)}{L(n s, \chi)}$ (resp. $\left.L(n s, \chi)^{-1}\right)$ for $\chi \in \operatorname{Hom}\left(C l_{F}, \mathbb{C}^{\times}\right)$and $0 \leq j \leq n-1$.

The proof of this Theorem is given in Section 4.4

Theorem 4.2.4 (Fourier expansion formula). For $\operatorname{Re}(s)>1$, we have

$$
\frac{E_{L,[\mathfrak{a}]}(z, s)}{\zeta_{F}\left(\mathfrak{a}^{-1}, n s\right)}=\sum_{j=0}^{n-2}\left(\Phi_{j}(z, s)+\Psi_{j}(z, s)\right)+\Phi_{n-1}(z, s) .
$$

In particular, $E_{L,[\mathfrak{a}]}(z, s)$ can be continued meromorphically to whole $s \in \mathbb{C}$

Proof. This follows from (4.6), Theorem 4.2.1, and Theorem 4.2.3.

Example 4.2.5. In the case where $F=\mathbb{Q}, n=2, L=\mathbb{Z} \oplus \mathbb{Z}$, and $\mathfrak{a}=\mathbb{Z}$, we have

$$
\begin{aligned}
& \Phi_{0}(z, s)=y_{1}^{s}, \quad \Phi_{1}(z, s)=\sqrt{\pi} y_{1}^{1-s} \frac{\Gamma\left(s-\frac{1}{2}\right)}{\Gamma(s)} \frac{\zeta_{\mathbb{Q}}(2 s-1)}{\zeta_{\mathbb{Q}}(2 s)}, \\
& \Psi_{0}(z, s)=\frac{2 \pi \sqrt{y_{1}}}{\Gamma(s) \zeta_{\mathbb{Q}}(2 s)} \sum_{n \in \mathbb{Z}, \neq 0}|n|^{s-\frac{1}{2}} \sigma_{1-2 s}(n) K_{s-\frac{1}{2}}\left(2 \pi|n| y_{1}\right) e^{2 \pi i n x_{12}},
\end{aligned}
$$

where $\sigma_{s}(n)$ is the usual divisor sum. In this case, Theorem 4.2.4 is nothing but the classical Fourier expansion of the real analytic Eisenstein series [8, Theorem 3.1.8].

\subsection{Proof of Theorem 4.2.1}

By Proposition 3.2 .3 (3), it suffices to compute the Fourier coefficients $\mathscr{I}_{\mathfrak{a} \hookrightarrow L, \nu}(z, s)$. Put $\bar{L}:=L^{(1)}, \bar{g}=\left(\bar{g}_{\sigma}\right)_{\sigma}:=g^{(1)}$ for simplicity.

We fix a fundamental domain of $N \backslash N(\mathbb{R})$. Set

$$
\begin{aligned}
L_{\mathfrak{a}, i} & :=\left\{x=\left(x_{j}\right)_{j} \in L_{\mathfrak{a}} \subset F^{n} \mid x_{j}=0(\forall j \leq i-1), x_{i} \neq 0\right\}, & & (i \leq n), \\
\bar{L}_{i} & :=\left\{x=\left(x_{j}\right)_{j} \in \bar{L} \subset F^{n-1} \mid x_{j}=0(\forall j \leq i-1), x_{i} \neq 0\right\}, & & (i \leq n-1), \\
N_{i}(\mathbb{R}) & :=\left\{\xi=\left(\xi_{1}, \ldots, \xi_{n-1}\right) \in N(\mathbb{R}) \mid \xi_{j}=0(j \neq i)\right\}, & & (i \leq n-1), \\
N^{i}(\mathbb{R}) & :=\left\{\xi=\left(\xi_{1}, \ldots, \xi_{n-1}\right) \in N(\mathbb{R}) \mid \xi_{i}=0\right\}, & & (i \leq n-1), \\
N_{i} & :=N \cap N_{i}(\mathbb{R}), \quad N^{i}:=N \cap N^{i}(\mathbb{R}), & & (i \leq n-1) .
\end{aligned}
$$


Here we identify matrices $\xi \in N(\mathbb{R})$ with vectors $\left(\xi_{1}, \ldots, \xi_{n-1}\right) \in F_{\infty}^{n-1}$ via the identification (4.3). We have $L_{\mathfrak{a}}=\coprod_{i=1}^{n} L_{\mathfrak{a}, i}, N=N_{i} N^{i}$, and if $i \leq n-1, N_{i}$ acts freely on $L_{\mathfrak{a}, i}$ by the matrix action from the right.

Step 1. (Decomposition of the integral) For $x \in F_{\infty}^{n}$, put $f(x):=\prod_{\sigma} \frac{\left|\operatorname{det} g_{\sigma}\right|^{n_{\sigma} s}}{\left\|x g_{\sigma} \mid\right\|^{n n_{\sigma} s}}$. Then we can decompose the integral $\mathscr{I}_{\mathfrak{a} \hookrightarrow L, \nu}(z, s)$ as

$$
\mathscr{I}_{\mathfrak{a} \hookrightarrow L, \nu}(z, s)=\frac{1}{\operatorname{vol}(N \backslash N(\mathbb{R}))} \sum_{i_{0}=1}^{n} \sum_{x \in \mathcal{O}_{F}^{\times} \backslash L_{\mathfrak{a}, i_{0}}} \int_{N \backslash N(\mathbb{R})} f(x \xi) e^{-2 \pi i\langle\nu, \xi\rangle} d \xi .
$$

Set $\mathscr{I}_{\nu, i_{0}}:=\sum_{x \in \mathcal{O}_{F}^{\times} \backslash L_{\mathfrak{a}, i_{0}}} \int_{N \backslash N(\mathbb{R})} f(x \xi) e^{-2 \pi i\langle\nu, \xi\rangle} d \xi$. In the case where $i_{0}=n$, we easily see that $\mathscr{I}_{\nu, n}=0$ unless $\nu=0$ and $[\mathfrak{a}]=\left[\mathfrak{a}_{n}\right]$, in which case we have

$$
\mathscr{I}_{\nu, n}=\operatorname{vol}(N \backslash N(\mathbb{R}))\left(\frac{N \mathfrak{a}}{N \mathfrak{a}_{n}}\right)^{n s} \prod_{\sigma}\left|\operatorname{det} \bar{g}_{\sigma}\right|^{n_{\sigma} s} .
$$

In the following, we assume $i_{0} \leq n-1$. Then we calculate as

$$
\begin{aligned}
\mathscr{I}_{\nu, i_{0}} & =\sum_{x \in \mathcal{O}_{F}^{\times} \backslash L_{\mathfrak{a}, i_{0}} / N_{i_{0}}} \sum_{\xi^{\prime} \in N_{i_{0}}} \int_{N \backslash N(\mathbb{R})} f\left(x \xi^{\prime} \xi\right) e^{-2 \pi i\left\langle\nu, \xi^{\prime} \xi\right\rangle} d \xi . \\
& =\sum_{x \in \mathcal{O}_{F}^{\times} \backslash L_{\mathfrak{a}, i_{0}} / N_{i_{0}}} \int_{N^{i_{0}} \backslash N^{i_{0}}(\mathbb{R})}\left(\int_{N_{i_{0}}(\mathbb{R})} f(x \xi) e^{-2 \pi i\langle\nu, \xi\rangle} d \xi_{i_{0}}\right) d \xi^{i_{0}},
\end{aligned}
$$

where $\xi^{\prime} \xi$ is the multiplication as matrices, and we use $e^{-2 \pi i\left\langle\nu, \xi^{\prime} \xi\right\rangle}=e^{-2 \pi i\langle\nu, \xi\rangle}$ since $\left\langle\nu, \xi^{\prime}\right\rangle \in \mathbb{Z}$. Furthermore, $d \xi_{i_{0}}$ and $d \xi^{i_{0}}$ are the Haar measures on $N_{i_{0}}(\mathbb{R})$ and $N^{i_{0}}(\mathbb{R})$ normalized in the same way as $d \xi$.

Step 2. (Calculation of the integral)

Now, $x \xi=\left(x_{1}, \ldots, x_{n-1}, x_{1} \xi_{1}+\cdots+x_{n-1} \xi_{n-1}+x_{n}\right)$ for $\xi=\left(\xi_{1}, \ldots, \xi_{n-1}\right)$. Since $x_{i_{0}} \neq 0$, we can replace $x_{1} \xi_{1}+\cdots+x_{n-1} \xi_{n-1}+x_{n}$ with $x_{i_{0}} \xi_{i_{0}}$ by the change of variables. Then we obtain

$$
\begin{aligned}
\int_{N^{i_{0}} \backslash N^{i_{0}}(\mathbb{R})} & \left(\int_{N_{i_{0}}(\mathbb{R})} f(x \xi) e^{-2 \pi i\langle\nu, \xi\rangle} d \xi_{i_{0}}\right) d \xi^{i_{0}} \\
=e^{2 \pi i \operatorname{Tr}_{F / \mathbb{Q}}\left(\frac{\nu_{i_{0}}}{x_{i_{0}}} x_{n}\right)} & \int_{N^{i_{0}} \backslash N^{i_{0}}(\mathbb{R})} e^{-2 \pi i T r_{F / \mathbb{Q}}\left(\xi_{1}\left(\nu_{1}-\frac{\nu_{i_{0}}}{x_{i_{0}}} x_{1}\right)+\cdots+\xi_{n-1}\left(\nu_{n-1}-\frac{\nu_{i_{0}}}{\left.\left.x_{i_{0}} x_{n-1}\right)\right)}\right.\right.} d \xi^{i_{0}} \\
& \times \int_{N_{i_{0}}(\mathbb{R})} f\left(x_{1}, \ldots, x_{n-1}, x_{i_{0}} \xi_{i_{0}}\right) e^{-2 \pi i \operatorname{Tr}_{F / \mathbb{Q}}\left(\nu_{i_{0}} \xi_{i_{0}}\right)} d \xi_{i_{0}}
\end{aligned}
$$

Here the first integral in (4.38) is 0 unless $\nu=\frac{\nu_{i_{0}}}{x_{i_{0}}}\left(x_{1}, \ldots, x_{n-1}\right)$, in which case equal to $\operatorname{vol}\left(N^{i_{0}} \backslash N^{i_{0}}(\mathbb{R})\right)$. Therefore we assume $\nu=\frac{\nu_{i_{0}}}{x_{i_{0}}}\left(x_{1}, \ldots, x_{n-1}\right)$. On the other hand, the 
second integral in (4.38) can be written as

$$
\prod_{\sigma}\left|\operatorname{det} \bar{g}_{\sigma}\right|^{n_{\sigma} s} \int_{F_{\sigma}} \frac{e^{-2 \pi i T r_{F_{\sigma} / \mathbb{R}}\left(\nu_{i_{0}} t_{\sigma}\right)}}{\left(|| \bar{x} \bar{g}_{\sigma} \|^{2}+\left|\bar{x} \mathbf{x}_{n, \sigma}+x_{i_{0}} t_{\sigma}\right|^{2}\right)^{n n_{\sigma} s / 2}} d t_{\sigma},
$$

where $\bar{x}=\left(x_{1}, \ldots, x_{n-1}\right)$ is the first $n-1$ components of $x$, and $\mathbf{x}_{n, \sigma} \in F_{\sigma}^{n-1}$ is the $\sigma$-component of $\mathbf{x}_{n}$, and $\bar{x} \mathbf{x}_{n, \sigma}$ is the scalar product. By an elementary computation, we have

$$
\begin{aligned}
& \int_{F_{\sigma}} \frac{e^{-2 \pi i \operatorname{Tr}_{F_{\sigma} / \mathbb{R}}\left(\nu_{i_{0}} t_{\sigma}\right)}}{\left(\left\|\bar{x} \bar{g}_{\sigma}\right\|^{2}+\left|\bar{x} \mathbf{x}_{n, \sigma}+x_{i_{0}} t_{\sigma}\right|^{2}\right)^{n n_{\sigma} s / 2}} d t_{\sigma} \\
& = \begin{cases}\left.n_{\sigma} \pi^{\frac{n_{\sigma}}{2}} \frac{\Gamma\left(\frac{n n_{\sigma} s-n_{\sigma}}{2}\right)}{\Gamma\left(\frac{n n_{\sigma} s}{2}\right)}\left|\sigma\left(x_{i_{0}}\right)\right|^{-n_{\sigma}}|| \bar{x} \bar{g}_{\sigma}\right|^{n_{\sigma}(1-n s)} & \left(\nu_{i_{0}}=0\right) \\
2^{n_{\sigma}} \pi^{\frac{n_{\sigma} n s}{2}} e^{2 \pi i T r_{F \sigma} / \mathbb{R}\left(\nu \mathbf{x}_{n, \sigma}\right)} \frac{K_{\underline{n n} \sigma s-n_{\sigma}}\left(2 \pi\left\|\nu \bar{g}_{\sigma}\right\|\right)}{\Gamma\left(\frac{n n_{\sigma} s}{2}\right)} \frac{\left|\sigma\left(\frac{\nu_{i_{0}}}{x_{i_{0}}}\right)\right|^{n_{\sigma}(n s-1)}}{\left|\sigma\left(x_{i_{0}}\right)\right|^{n_{\sigma}}}\left\|\nu \bar{g}_{\sigma}\right\|^{\frac{n_{\sigma}}{2}(1-n s)} & \left(\nu_{i_{0}} \neq 0\right)\end{cases}
\end{aligned}
$$

Step 3. (Calculation of the summation)

We take a closer look at $L_{\mathfrak{a}, i_{0}} / N_{i_{0}}$. For $x^{\prime}=\left(x_{1}, \ldots, x_{n-1}\right) \in F^{n-1}-\{0\}$, define

$$
\begin{aligned}
\mathfrak{b}_{x^{\prime}} & :=\left\{\alpha \in F \mid \alpha x_{i} \in \mathfrak{a}_{i}, 1 \leq \forall i \leq n-1\right\}, \\
S_{x^{\prime}} & :=\left\{\alpha \in F \mid\left(x_{1}, \ldots, x_{n-1}, \alpha\right) \in L_{\mathfrak{a}}\right\},
\end{aligned}
$$

so that we have $F x^{\prime} \cap \bar{L}=\mathfrak{b}_{x^{\prime}} x^{\prime}$ and $L_{\mathfrak{a}, i_{0}}=\coprod_{x^{\prime} \in \bar{L}_{i_{0}}}\left\{x^{\prime}\right\} \times S_{x^{\prime}}$. Note that $\mathfrak{b}_{x^{\prime}}$ is a fractional $\mathcal{O}_{F}$-ideal since $x^{\prime} \neq 0$, and we have, for any integral ideal $\mathfrak{m} \subset \mathcal{O}_{F}$,

$$
\left\{x^{\prime} \in \bar{L}-\{0\} \mid \mathfrak{a b}_{x^{\prime}}^{-1}=\mathfrak{m}\right\}=\bar{L}_{\mathfrak{a m}^{-1}},
$$

Lemma 4.3.1. We have $S_{x^{\prime}}=\emptyset$ unless $\mathfrak{a} \subset \mathfrak{b}_{x^{\prime}}$, in which case we have

(i) $S_{x^{\prime}}=\left\{\alpha \in \mathfrak{a}_{n} \mathfrak{a}^{-1} \mid v_{\mathfrak{p}}(\alpha)=v_{\mathfrak{p}}\left(\mathfrak{a}_{n} \mathfrak{a}^{-1}\right) \forall \mathfrak{p}\right.$ : prime ideal $\left.\mathfrak{p} \mid \mathfrak{a} \mathfrak{b}_{x^{\prime}}^{-1}\right\}$, where $v_{\mathfrak{p}}$ is the usual additive $\mathfrak{p}$-adic valuation. In particular, $\mathfrak{a}_{n} \mathfrak{b}_{x^{\prime}}^{-1}$ acts on $S_{x^{\prime}}$ by addition.

(ii) $S_{x^{\prime}} / \mathfrak{a}_{n} \mathfrak{b}_{x^{\prime}}^{-1}=\left(\mathfrak{a}_{n} \mathfrak{a}^{-1} / \mathfrak{a}_{n} \mathfrak{b}_{x^{\prime}}^{-1}\right)^{\times}$: the set of generators of $\mathfrak{a}_{n} \mathfrak{a}^{-1} / \mathfrak{a}_{n} \mathfrak{b}_{x^{\prime}}^{-1}$ as an $\mathcal{O}_{F} / \mathfrak{a} \mathfrak{b}_{x^{\prime}}^{-1}$ module.

(iii) $L_{\mathfrak{a}, i_{0}} / N_{i_{0}}=\coprod_{x^{\prime} \in \bar{L}_{i_{0}}}\left\{x^{\prime}\right\} \times\left(S_{x^{\prime}} / x_{i_{0}} \mathfrak{a}_{n} \mathfrak{a}_{i_{0}}^{-1}\right)$, where $x^{\prime}=\left(x_{1}, \ldots, x_{n-1}\right)$.

Proof. The first assertion and (i) follows from the fact

$$
\alpha \in S_{x^{\prime}} \Leftrightarrow \mathfrak{b}_{x^{\prime}} \cap \frac{1}{\alpha} \mathfrak{a}_{n}=\mathfrak{a} \Leftrightarrow \mathcal{O}_{F} \cap \frac{1}{\alpha} \mathfrak{a}_{n} \mathfrak{b}_{x^{\prime}}^{-1}=\mathfrak{a b}_{x^{\prime}}^{-1}, \quad \forall \alpha \in F^{\times} .
$$

(ii) follows directly from (i), and (iii) is obvious. 
Note that $\nu_{i_{0}}=0$ if and only if $\nu=0$ by the assumption $\nu=\frac{\nu_{i_{0}}}{x_{i_{0}}}\left(x_{1}, \ldots, x_{n-1}\right)$. Therefore, in the case $\nu=0$, we obtain

$$
\begin{gathered}
\mathscr{I}_{0, i_{0}}=\sum_{x^{\prime} \in \mathcal{O}_{F}^{\times} \backslash \bar{L}_{i_{0}}} \sum_{\alpha \in S_{x^{\prime}} / x_{i_{0}} \mathfrak{a}_{n} \mathfrak{a}_{i_{0}}^{-1}} \frac{\operatorname{vol}\left(N^{i_{0}} \backslash N^{i_{0}}(\mathbb{R})\right)}{\left|N_{F / \mathbb{Q}}\left(x_{i_{0}}\right)\right|} 2^{r_{2}(F)} \pi^{\frac{d}{2}} \frac{\Gamma_{F}(n s-1)}{\Gamma_{F}(n s)} \prod_{\sigma} \frac{\left|\operatorname{det} \bar{g}_{\sigma}\right|^{n_{\sigma} s}}{\|\left. x^{\prime} \bar{g}_{\sigma}\right|^{n_{\sigma}(n s-1)}} \\
=\frac{\operatorname{vol}(N \backslash N(\mathbb{R}))}{\sqrt{\left|d_{F}\right|}} 2^{r_{2}(F)} \pi^{\frac{d}{2}} \frac{\Gamma_{F}(n s-1)}{\Gamma_{F}(n s)}\left(\frac{N \mathfrak{a}}{N \mathfrak{a}_{n}}\right) \sum_{\substack{x^{\prime} \in \mathcal{O}_{F}^{\times} \backslash \bar{L}_{i_{0}} \\
\mathfrak{a b}_{x^{\prime}}^{-1} \subset \mathcal{O}_{F}}} \frac{\varphi\left(\mathfrak{a} \mathfrak{b}_{x^{\prime}}^{-1}\right)}{N\left(\mathfrak{a} \mathfrak{b}_{x^{\prime}}^{-1}\right)} \prod_{\sigma} \frac{\left|\operatorname{det} \bar{g}_{\sigma}\right|^{n_{\sigma} s}}{\left\|x^{\prime} \bar{g}_{\sigma}\right\|^{n_{\sigma}(n s-1)}},
\end{gathered}
$$

where we use

$$
\frac{\#\left(S_{x^{\prime}} / x_{i_{0}} \mathfrak{a}_{n} \mathfrak{a}_{i_{0}}^{-1}\right)}{\left|N_{F / \mathbb{Q}}\left(x_{i_{0}}\right)\right| \operatorname{vol}\left(N_{i_{0}} \backslash N_{i_{0}}(\mathbb{R})\right)}=\frac{\varphi\left(\mathfrak{a b}_{x^{\prime}}^{-1}\right)}{N\left(\mathfrak{a}_{n} \mathfrak{b}_{x^{\prime}}^{-1}\right) \sqrt{\left|d_{F}\right|}} .
$$

By taking the summation over $1 \leq i_{0} \leq n-1$, and using (4.43) and Proposition 3.2.3 (3), we obtain Theorem 4.2.1 (1).

Similarly, in the cases where $\nu \neq 0$, we obtain

$$
\begin{aligned}
\mathscr{I}_{\nu, i_{0}}= & \operatorname{vol}(N \backslash N(\mathbb{R})) 2^{d} \pi^{\frac{d n s}{2}} e^{2 \pi i\left\langle\nu, \mathbf{x}_{n}\right\rangle} \frac{K_{F}\left(n s-1,\left(2 \pi|| \nu \bar{g}_{\sigma}||\right)_{\sigma}\right)}{\Gamma_{F}(n s)} \prod_{\sigma} \frac{\left|\operatorname{det} \bar{g}_{\sigma}\right|^{n_{\sigma} s}}{\| \nu \bar{g}_{\sigma}||^{\frac{n_{\sigma}}{2}(n s-1)}} \\
& \times \sum_{\substack{x^{\prime} \in \mathcal{O}_{F}^{\times} \backslash \bar{L}_{i_{0}} \\
\nu=\frac{\nu_{i_{0}}}{x_{i_{0}}} x^{\prime}}} \frac{\left|N_{F / \mathbb{Q}}\left(\frac{\nu_{i_{0}}}{x_{i_{0}}}\right)\right|^{n s-1}}{\left|N_{F / \mathbb{Q}}\left(x_{i_{0}}\right)\right| \operatorname{vol}\left(N_{i_{0}} \backslash N_{i_{0}}(\mathbb{R})\right)} \sum_{\alpha \in S_{x^{\prime}} / x_{i_{0}} \mathfrak{a}_{n} \mathfrak{a}_{i_{0}}^{-1}} e^{2 \pi i T r_{F / \mathbb{Q}}\left(\frac{\nu_{i_{0}}}{x_{i_{0}}} \alpha\right)} .
\end{aligned}
$$

Now, suppose $\nu \in\left(N^{\vee}\right)_{\mathfrak{n}^{-1}}$ for an integral ideal $\mathfrak{n} \subset \mathcal{O}_{F}$ (and $\left.\nu_{i_{0}} \neq 0\right)$. Then we claim that the last summation in (4.47) is 0 unless $\mathfrak{a b}_{x^{\prime}}^{-1} \subset \mathcal{O}_{F}$, in which case we have

$$
\sum_{\alpha \in S_{x^{\prime}} / x_{i_{0}} \mathfrak{a}_{n} \mathfrak{a}_{i_{0}}^{-1}} e^{2 \pi i T r_{F / \mathbb{Q}}\left(\frac{\nu_{i_{0}}}{x_{i_{0}}} \alpha\right)}=\#\left(\mathfrak{a}_{n} \mathfrak{b}_{x^{\prime}}^{-1} / x_{i_{0}} \mathfrak{a}_{n} \mathfrak{a}_{i_{0}}^{-1}\right) \tau\left(\mathfrak{a} \mathfrak{b}_{x^{\prime}}^{-1}, \mathfrak{n} \mathfrak{d}_{F}^{-1}\right) .
$$

Indeed, by (4.43), we have $\left(N^{\vee}\right)_{\mathfrak{n}^{-1}}=\left\{\nu \in N^{\vee} \mid \mathfrak{a}_{n} \mathfrak{b}_{\nu}^{-1} \mathfrak{d}_{F}=\mathfrak{n}\right\}$, and therefore, for $x^{\prime} \in \bar{L}_{i_{0}}$ such that $\nu=\frac{\nu_{i_{0}}}{x_{i_{0}}} x^{\prime}$, we have

$$
\frac{\nu_{i_{0}}}{x_{i_{0}}} \mathfrak{a}_{n} \mathfrak{b}_{x^{\prime}}^{-1}=\mathfrak{a}_{n} \mathfrak{b}_{\nu}^{-1}=\mathfrak{n} \mathfrak{d}_{F}^{-1} \subset \mathfrak{d}_{F}^{-1} .
$$

Now, the claim follows easily from Lemma 4.3.1. Thus, for $\nu \in\left(N^{\vee}\right)_{\mathfrak{n}^{-1}}$, we obtain $\mathscr{I}_{\nu, i_{0}}=0$ unless $\nu_{i_{0}} \neq 0$ and $\nu_{j}=0$ for all $j<i_{0}$, in which case we have

$$
\begin{aligned}
& \mathscr{I}_{\nu, i_{0}}=\frac{\operatorname{vol}(N \backslash N(\mathbb{R}))}{\sqrt{\left|d_{F}\right|}} 2^{d} \pi^{\frac{d n s}{2}} e^{2 \pi i\left\langle\nu, \mathbf{x}_{n}\right\rangle} \frac{K_{F}\left(n s-1,\left(2 \pi|| \nu \bar{g}_{\sigma} \|\right)_{\sigma}\right)}{\Gamma_{F}(n s)} \prod_{\sigma} \frac{\left|\operatorname{det} \bar{g}_{\sigma}\right|^{n_{\sigma} s}}{\left\|\nu \bar{g}_{\sigma}\right\|^{\frac{n_{\sigma}}{2}(n s-1)}} \\
& \times\left(\frac{N \mathfrak{a}}{N \mathfrak{a}_{n}}\right)^{n s} N\left(\mathfrak{n} \mathfrak{d}_{F}^{-1}\right)^{n s-1} \sum_{\substack{\mathfrak{m} \in\left[\mathfrak{a a}_{n}^{-1} \mathfrak{n d}_{F}^{-1}\right] \\
\mathfrak{m} \subset \mathcal{O}_{F}}} \frac{\tau\left(\mathfrak{m}, \mathfrak{n} \mathfrak{d}_{F}^{-1}\right)}{N \mathfrak{m}^{n s}} \text {. }
\end{aligned}
$$


Here we use the identity

$$
\begin{aligned}
\left\{x^{\prime} \in \mathcal{O}_{F}^{\times} \backslash \bar{L}_{i_{0}} \mid \nu=\frac{\nu_{i_{0}}}{x_{i_{0}}} x^{\prime}, \mathfrak{a} \mathfrak{b}_{x^{\prime}}^{-1}=\mathfrak{m}\right\} \stackrel{i_{0} \text {-th proj. }}{\stackrel{\sim}{\sim}}\left\{x_{i_{0}} \in \mathcal{O}_{F}^{\times} \backslash\left(\mathfrak{a}_{i_{0}}-\{0\}\right) \mid \frac{x_{i_{0}}}{\nu_{i_{0}}} \mathfrak{a b}_{\nu}^{-1}=\mathfrak{m}\right\} \\
=\quad\left\{x_{i_{0}} \in \mathcal{O}_{F}^{\times} \backslash F^{\times} \mid \frac{x_{i_{0}}}{\nu_{i_{0}}} \mathfrak{a b}_{\nu}^{-1}=\mathfrak{m}\right\},
\end{aligned}
$$

for any integral ideal $\mathfrak{m} \in\left[\mathfrak{a a}_{n}^{-1} \mathfrak{n} \mathfrak{d}_{F}^{-1}\right]$. By taking the summation over $1 \leq i_{0} \leq n-1$ and $\nu \in N^{\vee}-\{0\}$, we obtain Theorem 4.2.1 (2). This completes the proof.

\subsection{Proof of Theorem 4.2 .3}

The assertion for $\Phi_{j}(z, s)$ follows directly from Corollary 4.1.5. To prove the assertion for $\Psi_{j}(z, s)$, again by Corollary 4.1.5, we rewrite $\Psi_{j}(z, s)$ as

$$
\begin{aligned}
& \Psi_{j}(z, s)=d_{j}(z, s)\left(N \mathfrak{d}_{F}^{-1}\right)^{n s-j-1} \frac{1}{h_{F}} \sum_{\chi \in \operatorname{Hom}\left(C l_{F}, \mathbb{C}^{\times}\right)} \frac{\chi\left(\mathfrak{a}^{-1} \mathfrak{a}_{n-j} \mathfrak{d}_{F}\right)}{L(n s, \chi)} \sum_{\mathscr{A} \in C l_{F}} \chi\left(\mathscr{A}^{-1}\right) \\
\times & \sum_{\substack{\mathfrak{n} \in \mathscr{A}^{\prime} \\
\mathfrak{n} \subset \mathcal{O}_{F}}} \sum_{\substack{\nu \in(\Lambda(j+1))_{\mathfrak{n}}-1\\
}}(N \mathfrak{n})^{n s-j-1} \sigma_{1+j-n s}(\mathfrak{n}, \chi) \frac{K_{F}\left(n s-j-1,\left(2 \pi\left\|\nu g_{\sigma}^{(j+1)}\right\|\right)_{\sigma}\right)}{\prod_{\sigma}\left\|\nu g_{\sigma}^{(j+1)}\right\|^{\frac{n_{\sigma}}{2}(n s-j-1)}} e^{2 \pi i\left\langle\nu, \mathbf{x}_{n-j}\right\rangle} .
\end{aligned}
$$

Therefore, it suffices to prove that the second row of (4.52) converges absolutely and compactly for $s \in \mathbb{C}$. We use the following asymptotic formulas.

Lemma 4.4.1. Let $R$ be any real number such that $R>1$.

(1) For any integral ideal $\mathfrak{n}$, any $\chi \in \operatorname{Hom}\left(C l_{F}, \mathbb{C}^{\times}\right)$, and any $s \in \mathbb{C}$ such that $|\operatorname{Re}(s)| \leq$ $R$, we have

$$
\left|\sigma_{s}(\mathfrak{n}, \chi)\right| \leq(N \mathfrak{n})^{2 R}
$$

(2) There exists $C>0$ such that for any $x \in \mathbb{R}_{>0}$ and any $s \in \mathbb{C}$ such that $|\operatorname{Re}(s)| \leq R$, we have

$$
\left|K_{s}(x) x^{-s}\right| \leq C e^{-\frac{1}{2} x} x^{-10 R} .
$$

(3) There exists $C>0$ such that for any $x=\left(x_{\sigma}\right)_{\sigma} \in T_{F}$, we have

$$
\sum_{u \in U_{F}} \prod_{\sigma \in S_{F}} e^{-x_{\sigma} u_{\sigma}} \leq C N_{F / \mathbb{Q}}(x)^{-\frac{1}{4}} .
$$

See Section 2.1 for the definition of $U_{F} \subset T_{F}$. 
Proof. (1) is elementary and we omit the proof. To prove (2) we use the following well-known asymptotic behavior of the $K$-Bessel function:

$$
\lim _{x \rightarrow \infty} \sqrt{x} K_{s}(x) e^{x}=\sqrt{\frac{\pi}{2}}, \quad \lim _{x \rightarrow 0} K_{s}(x) x^{s}=2^{s-1} \Gamma(s), \quad(s \in \mathbb{C}, \operatorname{Re}(s)>0)
$$

Now, since $\left|K_{s}(x) x^{-s}\right| \leq K_{\operatorname{Re}(s)}(x) x^{-\operatorname{Re}(s)}$, we may assume $-R \leq s \leq R$. Then, by the integral expression (4.17) of $K_{s}(x)$, we have

$$
\begin{aligned}
K_{s}(x) & \leq \frac{1}{2} \int_{0}^{1} e^{-\frac{1}{2} x\left(u+\frac{1}{u}\right)} u^{-R} \frac{d u}{u}+\frac{1}{2} \int_{1}^{\infty} e^{-\frac{1}{2} x\left(u+\frac{1}{u}\right)} u^{R} \frac{d u}{u} \\
& \leq K_{-R}(x)+K_{R}(x)=2 K_{R}(x) .
\end{aligned}
$$

Therefore, we have

$$
K_{s}(x) x^{-s} e^{x / 2} x^{10 R} \leq 2 K_{R}(x) x^{10 R-s} e^{x / 2} .
$$

By the asymptotic formulas, the right hand side can be bounded uniformly in $s$ for $-R \leq s \leq R$. This proves (2). To prove (3), first observe that

$$
\begin{aligned}
\sum_{\sigma} x_{\sigma} u_{\sigma} & \geq \sum_{\substack{\sigma \\
x_{\sigma} u_{\sigma} \geq 1}} \log \left(x_{\sigma} u_{\sigma}\right) \geq \frac{1}{4} \sum_{\substack{\sigma \\
x_{\sigma} u_{\sigma} \geq 1}} 2 n_{\sigma} \log \left(x_{\sigma} u_{\sigma}\right) \\
& =\frac{1}{4}\left(\log \left(N_{F / \mathbb{Q}}(x)\right)+\sum_{\sigma} n_{\sigma}\left|\log \left(x_{\sigma} u_{\sigma}\right)\right|\right),
\end{aligned}
$$

where we use $N_{F / \mathbb{Q}}(u)=1$. Thus we have

$$
\sum_{u \in U_{F}} \prod_{\sigma} e^{-x_{\sigma} u_{\sigma}} \leq N_{F / \mathbb{Q}}(x)^{-\frac{1}{4}} \sum_{u \in U_{F}} \prod_{\sigma} e^{-\frac{1}{4}\left(n_{\sigma}\left|\log \left(u_{\sigma}\right)+\log \left(x_{\sigma}\right)\right|\right)} .
$$

Then, we easily see that there exists $C>0$ which is independent of $x$ such that

$$
\sum_{u \in U_{F}} \prod_{\sigma} e^{-\frac{1}{4}\left(n_{\sigma}\left|\log \left(u_{\sigma}\right)+\log \left(x_{\sigma}\right)\right|\right)} \leq C,
$$

using the fact that $U_{F}$ is a lattice in $T_{F / \mathbb{Q}}$. This proves (3).

Let $R>n \geq 1$. Then, by Lemma 4.4.1, there exist $C_{1}, C_{2}>0$ such that for $|\operatorname{Re}(n s-j-1)| \leq R$, the second row of (4.52) can be bounded as

$$
\begin{aligned}
& \sum_{\mathfrak{n} \in \mathscr{A}} \sum_{\nu \in\left(\Lambda^{(j+1)}\right)_{\mathfrak{n}-1}}\left|(N \mathfrak{n})^{n s-j-1} \sigma_{1+j-n s}(\mathfrak{n}, \chi) \prod_{\sigma} \frac{K_{\frac{n_{\sigma}}{2}(n s-j-1)}\left(2 \pi\left\|\nu g_{\sigma}^{(j+1)}\right\|\right)}{\left\|\nu g_{\sigma}^{(j+1)}\right\|^{\frac{n_{\sigma}}{2}(n s-j-1)}}\right| \\
\leq & C_{1} \sum_{\mathfrak{n} \in \mathscr{A}} \sum_{\nu \in\left(\Lambda^{(j+1)}\right)_{\mathfrak{n}-1}}(N \mathfrak{n})^{3 R} \prod_{\sigma}\left\|\nu g_{\sigma}^{(j+1)}\right\|^{-5 n_{\sigma} R} e^{-\pi\left\|\nu g_{\sigma}^{(j+1)}\right\|} \\
\leq & C_{1} \sum_{\mathfrak{n} \in \mathscr{A}} \sum_{u \in \mathcal{O}_{F}^{\times}} \sum_{\nu \in \mathcal{O}_{F}^{\times} \backslash\left(\Lambda^{(j+1)}\right)_{\mathfrak{n}-1}}(N \mathfrak{n})^{3 R} \prod_{\sigma}\left\|u \nu g_{\sigma}^{(j+1)}\right\|^{-5 n_{\sigma} R} e^{-\pi\left\|u \nu g_{\sigma}^{(j+1)}\right\|} \\
\leq & C_{2} \sum_{\mathfrak{n} \in \mathscr{A}} \sum_{\nu \in \mathcal{O}_{F}^{\times} \backslash\left(\Lambda^{(j+1)}\right)_{\mathfrak{n}}-1}(N \mathfrak{n})^{3 R} \prod_{\sigma}\left\|\nu g_{\sigma}^{(j+1)}\right\|^{-5 n_{\sigma} R-\frac{1}{4} n_{\sigma} .}
\end{aligned}
$$


Let us fix $\mathfrak{n} \in \mathscr{A}$. Then, (4.67) can be rewritten as

$$
\begin{aligned}
& C_{3} \sum_{x \in \mathcal{O}_{F}^{\times} \backslash \mathfrak{n}^{-1}}\left|N_{F / \mathbb{Q}}(x)\right|^{3 R-5 R-\frac{1}{4}} \sum_{\substack{\nu \in \mathcal{O}_{F}^{\times} \backslash\left(\Lambda^{(j+1)}\right)_{\mathfrak{n}}-1\\
}} \prod_{\sigma}\left\|\nu g_{\sigma}^{(j+1)}\right\|^{-n_{\sigma}\left(5 R+\frac{1}{4}\right)} \\
= & C_{3}(N \mathfrak{n})^{2 R+\frac{1}{4}} \zeta_{F}\left(\mathfrak{n}^{-1}, 2 R+\frac{1}{4}\right) \sum_{\nu \in \mathcal{O}_{F}^{\times} \backslash\left(\Lambda^{(j+1)}\right)_{\mathfrak{n}-1}} \prod_{\sigma}\left\|\nu g_{\sigma}^{(j+1)}\right\|^{-n_{\sigma}\left(5 R+\frac{1}{4}\right)},
\end{aligned}
$$

where $C_{3}:=C_{2}(N \mathfrak{n})^{3 R}$. By Proposition 3.2 .3 (1) applied to the parabolic data $\mathfrak{n}^{-1} \hookrightarrow$ $\Lambda^{(j+1)}$, the last infinite series converges. This completes the proof of Theorem 4.2.3.

\section{$5 \quad$ Residue formula and Kronecker's limit formula}

In this section, we compute the residue and the constant term of our Eisenstein series $E_{L,[\mathfrak{a}]}(z, s)$ and the "relative" partial zeta function $\zeta_{E / F,[\mathfrak{a}]}\left(\mathfrak{A}^{-1}, s\right)$ at $s=1$.

We keep the notations in Section 4 . That is, $F$ is a number field of degree $d, L \subset F^{n}$ $(n \geq 2)$ is an $\mathcal{O}_{F}$-lattice of the form $L=\mathfrak{a}_{1} \oplus \cdots \oplus \mathfrak{a}_{n}$, and $E_{L,[\mathfrak{a}]}(z, s)$ is the Eisenstein series associated to a parabolic data $\mathfrak{a} \hookrightarrow L$, etc. In the following, for any meromorphic function $f$ on $\mathbb{C}$, and $a \in \mathbb{C}$, we denote by $f^{(-1)}(a)$ (resp. $f^{(0)}(a)$ ) the residue (resp. constant term) of $f(s)$ at $s=a$.

\subsection{Residue formula for $E_{L,[\mathfrak{a}]}(z, s)$}

Recall that by the Fourier expansion formula (Theorem 4.2.4), we have

$$
\frac{E_{L,[\mathfrak{a}]}(z, s)}{\zeta_{F}\left(\mathfrak{a}^{-1}, n s\right)}=\sum_{j=0}^{n-2}\left(\Phi_{j}(z, s)+\Psi_{j}(z, s)\right)+\Phi_{n-1}(z, s) .
$$

Then, by Theorem 4.2.3, $\sum_{j=0}^{n-2}\left(\Phi_{j}(z, s)+\Psi_{j}(z, s)\right)$ is holomorphic at $s=1$. On the other hand, by Corollary 4.1.5, we have

$$
\Phi_{n-1}(z, s)=c_{n-1}(z, s) \frac{1}{h_{F}} \sum_{\chi \in \operatorname{Hom}\left(C l_{F}, \mathbb{C}^{\times}\right)} \chi\left(\left[\mathfrak{a}_{1} \mathfrak{a}^{-1}\right]\right) \frac{L(n s-n+1, \chi)}{L(n s, \chi)} .
$$

Therefore we get the following.

Theorem 5.1.1 (Residue formula for $\left.E_{L,[\mathfrak{a}]}(z, s)\right)$. The Eisenstein series $E_{L,[\mathfrak{a}]}(z, s)$ has a simple pole at $s=1$, and we have

$$
\begin{aligned}
& E_{L,[\mathfrak{a}]}^{(-1)}(z, 1)=c_{n-1}(z, 1) \frac{\kappa_{F}}{n h_{F}} \frac{\zeta_{F}\left(\mathfrak{a}^{-1}, n\right)}{\zeta_{F}(n)} \\
& =\left(N \mathfrak{a}_{1} \cdots N \mathfrak{a}_{n}\right)^{-1}\left(\frac{2^{r_{2}(F)} \pi^{\frac{d}{2}}}{\sqrt{\left|d_{F}\right|}}\right)^{n} \frac{2^{r_{1}(F)}}{\Gamma_{F}(n)} \frac{R_{F}}{n w_{F}} \frac{\zeta_{F}\left(\mathfrak{a}^{-1}, n\right)}{\zeta_{F}(n)}
\end{aligned}
$$

where $\zeta_{F}(s)$ is the Dedekind zeta function of $F$, and $\kappa_{F}=\zeta_{F}^{(-1)}(1)$ is its residue at $s=1$. 
Remark. Note that $c_{n-1}(z, 1)$ and hence $E_{L,[\mathfrak{a}]}^{(-1)}(z, 1)$ are independent of $z$. We put $\mathbf{c}_{n-1}:=c_{n-1}(z, 1)$ for simple.

Proof. This follows from the fact that $L(s, \chi)$ is holomorphic at $s=1$ unless $\chi$ is trivial, in which case $L(s, \chi)=\zeta_{F}(s)$ has a simple pole.

\subsection{Kronecker's limit formula for $E_{L,[\mathfrak{a}]}(z, s)$}

First recall the classical case where $F=\mathbb{Q}, n=2, L=\mathbb{Z} \oplus \mathbb{Z}$, and $\mathfrak{a}=\mathbb{Z}$ (cf. Example 4.2.5). In this case, we have

$$
\begin{aligned}
\Phi_{0}^{(0)}(z, 1) & +\Psi_{0}^{(0)}(z, 1)=\Phi_{0}(z, 1)+\Psi_{0}(z, 1)=-\frac{6}{\pi} \log |\eta(z)|^{2} \\
\Phi_{1}^{(0)}(z, 1) & =\frac{6}{\pi}\left(\gamma+\frac{1}{2}\left(\psi\left(\frac{1}{2}\right)-\psi(1)-\log y_{1}-2 \frac{\zeta_{\mathbb{Q}}^{(1)}(2)}{\zeta_{\mathbb{Q}}(2)}\right)\right) \\
& =\frac{6}{\pi}\left(\gamma-\log 2-\log \sqrt{y_{1}}\right)-\frac{6}{\pi} \frac{\zeta_{\mathbb{Q}}^{(1)}(2)}{\zeta_{\mathbb{Q}}(2)},
\end{aligned}
$$

where $\eta(z)$ is the Dedekind eta function, $\gamma=\zeta_{\mathbb{Q}}^{(0)}(1)$ is Euler's constant, and $\psi(s):=\frac{\Gamma^{\prime}(s)}{\Gamma(s)}$ is the digamma function, that is, the logarithmic derivative of the gamma function.

Definition 5.2.1. (1) We define

$$
H_{L,[\mathfrak{a}]}(z):=\zeta_{F}\left(\mathfrak{a}^{-1}, n\right) \sum_{j=0}^{n-2}\left(\Phi_{j}(z, 1)+\Psi_{j}(z, 1)\right), \quad \text { for } z \in \mathfrak{h}_{F}^{n} .
$$

(2) Let $k / \mathbb{Q}$ be a number field. For a character $\chi \in \operatorname{Hom}\left(C l_{k}, \mathbb{C}^{\times}\right)$, let $L(s, \chi)$ be the Hecke $L$-function associated to the character $\chi$. Then we set

$$
\gamma_{k}(\chi):=L^{(0)}(1, \chi) \text {. }
$$

Moreover, let $\Gamma_{k}(s)$ be the gamma function over $k$ (4.16). We define the digamma function $\psi_{k}(s)$ over $k$ as

$$
\psi_{k}(s):=\frac{d}{d s} \log \Gamma_{k}(s)=\frac{\Gamma_{k}^{\prime}(s)}{\Gamma_{k}(s)}=\sum_{\sigma \in S_{k}} \frac{n_{\sigma}}{2} \psi\left(\frac{n_{\sigma} s}{2}\right) .
$$

Theorem 5.2.2 (Kronecker's limit formula for $\left.E_{L,[\mathfrak{a}]}(z, s)\right)$. We have

$$
\begin{array}{r}
E_{L,[\mathfrak{a}]}^{(0)}(z, 1)=H_{L,[\mathfrak{a}]}(z)+\mathbf{c}_{n-1} \\
\frac{1}{h_{F}} \sum_{\chi \in \operatorname{Hom}\left(C l_{F}, \mathbb{C}^{\times}\right)} \chi\left(\left[\mathfrak{a}_{1} \mathfrak{a}^{-1}\right]\right) \frac{\zeta_{F}\left(\mathfrak{a}^{-1}, n\right)}{L(n, \chi)} \gamma_{F}(\chi) \\
+\mathbf{c}_{n-1} \frac{\kappa_{F}}{h_{F}} \frac{\zeta_{F}\left(\mathfrak{a}^{-1}, n\right)}{\zeta_{F}(n)}\left(\psi_{F}(1)-\psi_{F}(n)-\log \left(N \mathfrak{a}_{1}\right)-\sum_{k=1}^{n-1} \frac{n-k}{n} \log \left(N_{F / \mathbb{Q}}\left(y_{k}\right)\right)\right) \\
+\mathbf{c}_{n-1} \frac{\kappa_{F}}{h_{F}} \frac{\zeta_{F}\left(\mathfrak{a}^{-1}, n\right)}{\zeta_{F}(n)}\left(\frac{\zeta_{F}^{(1)}\left(\mathfrak{a}^{-1}, n\right)}{\zeta_{F}\left(\mathfrak{a}^{-1}, n\right)}-\frac{\zeta_{F}^{(1)}(n)}{\zeta_{F}(n)}\right)
\end{array}
$$


Proof. This follows from the Fourier expansion formula (5.1) (Theorem 4.2.4), (5.2), and Theorem 5.1.1.

Corollary 5.2.3 (Automorphy of the function $\left.H_{L,[a]}\right)$. The function

$$
H_{L,[\mathfrak{a}]}^{*}(z):=H_{L,[\mathfrak{a}]}(z)-\mathbf{c}_{n-1} \frac{\kappa_{F}}{h_{F}} \frac{\zeta_{F}\left(\mathfrak{a}^{-1}, n\right)}{\zeta_{F}(n)} \sum_{k=1}^{n-1} \frac{n-k}{n} \log \left(N_{F / \mathbb{Q}}\left(y_{k}\right)\right)
$$

is an automorphic function on $\mathfrak{h}_{F}^{n}$, that is, we have $H_{L,[\mathfrak{a}]}^{*}(\gamma z)=H_{L,[\mathfrak{a}]}^{*}(z)$ for all $\gamma \in \Gamma_{L}$.

Proof. Since $E_{L,[\mathfrak{a}]}(z, s)$ is an automorphic function (Proposition 3.2 .3$), E_{L,[\mathfrak{a}]}^{(0)}(z, 1)$ is also an automorphic function on $\mathfrak{h}_{F}^{n}$. Then, by Theorem $\left[5.2 .2\right.$, we obtain $H_{L,[\mathfrak{a}]}^{*}(\gamma z)-$ $H_{L,[\mathfrak{a}]}^{*}(z)=E_{L,[\mathfrak{a}]}^{(0)}(\gamma z, 1)-E_{L,[\mathfrak{a}]}^{(0)}(z, 1)=0$ for all $\gamma \in \Gamma_{L}$.

Remark. By the identity (5.4), we see that the function $H_{L,[\mathfrak{a}]}(z)$ gives a generalization of the function $-\frac{6}{\pi} \log |\eta(z)|^{2}$. In fact, in the case where $n=2$, the $\operatorname{sum} \sum_{\mathscr{A} \in C l_{F}} H_{L, \mathscr{A}}(z)$ coincides with the function $h_{F}(z, \mathfrak{a}, \mathfrak{b})$ considered by Yamamoto [18, Theorem 2.5.1] up to some constant factors, and Corollary 5.2 .3 gives a generalization of [18, Corollary $2.5 .2]$.

\subsection{Application to $\zeta_{E / F,[\mathfrak{a}]}\left(\mathfrak{A}^{-1}, s\right)$}

Let the notations be as in Section 2.1 and Section 3.3. That is, $E / F$ is an extension of number fields of degree $n \geq 2$, and $\mathfrak{A} \subset E$ is a fractional $\mathcal{O}_{E}$-ideal. In this section, we take a basis $w:={ }^{t}\left(w_{1}, \ldots, w_{n}\right)$ of $E$ over $F$ so that the lattice $L \subset F^{n}$ corresponding to $\mathfrak{A}$ via the isomorphism $w: F^{n} \stackrel{\sim}{\rightarrow} E$ is of the form $L=\mathfrak{a}_{1} \oplus \cdots \oplus \mathfrak{a}_{n}$ for some anti-integral $\mathcal{O}_{F}$-ideals $\mathfrak{a}_{i}(1 \leq i \leq n)$. Note that this is always possible by Proposition 2.2.5. Then, $\varpi: T_{E / F} \rightarrow \mathfrak{h}_{F}^{n}$ is the Heegner object associated to $w$. Let $\mathfrak{a}$ be an anti-integral $\mathcal{O}_{F}$-ideal, and let $E_{L,[\mathfrak{a}]}(z, s)$ be the Eisenstein series associated to the parabolic data $\mathfrak{a} \hookrightarrow L$.

Combining Theorem 3.3.3. Theorem 4.2.4, Theorem 5.1.1, and Theorem 5.2.2, we obtain the following properties of $\zeta_{E / F,[\mathfrak{a}]}\left(\mathfrak{A}^{-1}, s\right)$.

Theorem 5.3.1. (1) The relative partial zeta function $\zeta_{E / F,[\mathfrak{a}]}\left(\mathfrak{A}^{-1}, s\right)$ can be continued meromorphically to whole $s \in \mathbb{C}$, and has a simple pole at $s=1$.

(2) (Residue formula) We have

$$
\begin{aligned}
\zeta_{E / F,[\mathfrak{a}]}^{(-1)}\left(\mathfrak{A}^{-1}, 1\right) & =\frac{2^{r_{1}(E)}(2 \pi)^{r_{2}(E)} R_{E}}{w_{E} \sqrt{\left|d_{E}\right|}} \frac{\zeta_{F}\left(\mathfrak{a}^{-1}, n\right)}{\zeta_{F}(n)} \\
& =\frac{\kappa_{E}}{h_{E}} \frac{\zeta_{F}\left(\mathfrak{a}^{-1}, n\right)}{\zeta_{F}(n)}
\end{aligned}
$$

where $\kappa_{E}:=\zeta_{E}^{(-1)}(1)$ is the residue of the Dedekind zeta function $\zeta_{E}(s)$ of $E$. 
(3) (Kronecker's limit formula) We have

$$
\begin{array}{r}
\zeta_{E / F,[\mathfrak{a}]}^{(0)}\left(\mathfrak{A}^{-1}, 1\right)=n \mathbf{c}_{n-1}^{-1} \frac{h_{F}}{h_{E}} \frac{\kappa_{E}}{\kappa_{F}}\left(\frac{1}{R_{E / F}} \int_{T_{E / F} / U_{E / F}} H_{L,[\mathfrak{a}]}^{*}(\bar{\varpi}(t)) d^{\times} t_{E / F}\right. \\
\left.+\frac{1}{h_{F}} \sum_{\chi} \chi\left(\left[\mathfrak{a}_{1} \mathfrak{a}^{-1}\right]\right) \frac{\zeta_{F}\left(\mathfrak{a}^{-1}, n\right)}{L(n, \chi)} \gamma_{F}(\chi)\right) \\
+\frac{\kappa_{E}}{h_{E}} \frac{\zeta_{F}\left(\mathfrak{a}^{-1}, n\right)}{\zeta_{F}(n)}\left(\left(n r_{F}-r_{E}\right) \log 2+\log \frac{\left|d_{E}\right|^{\frac{1}{2}}}{\left|d_{F}\right|^{\frac{n}{2}}}+\log \frac{N \mathfrak{a}_{1}^{n}}{N \mathfrak{a}_{1} \cdots N \mathfrak{a}_{n}}\right. \\
\left.-n \frac{\zeta_{F}^{(1)}\left(\mathfrak{a}^{-1}, n\right)}{\zeta_{F}\left(\mathfrak{a}^{-1}, n\right)}+n \frac{\zeta_{F}^{(1)}(n)}{\zeta_{F}(n)}\right) .
\end{array}
$$

Proof. (1) follows from Theorem 3.3.3 and Theorem 4.2.4. To prove (2) and (3), we use the following identity:

$$
\left|\Delta_{w}\right|^{\frac{1}{2}}\left(=\left|N_{F / \mathbb{Q}}(\operatorname{det} W)\right|\right)=\frac{2^{n r_{2}(F)-r_{2}(E)}\left|d_{E}\right|^{\frac{1}{2}} N \mathfrak{A}}{\left|d_{F}\right|^{\frac{n}{2}} N \mathfrak{a}_{1} \cdots N \mathfrak{a}_{n}} .
$$

Indeed, by the definition of $W$ and the normalization of the Haar measures $d x_{E}, d x_{F}$ (Section 3.1), we see

$$
\begin{aligned}
\left|d_{E}\right|^{\frac{1}{2}} N \mathfrak{A} & =\operatorname{vol}\left(E_{\infty} / \mathfrak{A}\right)=2^{r_{2}(E)-n r_{2}(F)}\left|\Delta_{w}\right|^{\frac{1}{2}} \operatorname{vol}\left(F_{\infty}^{n} / \mathfrak{a}_{1} \oplus \cdots \oplus \mathfrak{a}_{n}\right) \\
& =2^{r_{2}(E)-n r_{2}(F)}\left|\Delta_{w}\right|^{\frac{1}{2}}\left|d_{F}\right|^{\frac{n}{2}} N \mathfrak{a}_{1} \cdots N \mathfrak{a}_{n}
\end{aligned}
$$

Then, (2) and (3) follows from Theorem5.1.1 and Theorem 5.2.2 combined with Theorem 3.3 .3 .

\section{Acknowledgements}

I would like to express my deepest gratitude to Professor Takeshi Tsuji for the constant encouragement and valuable comments during the study. I would also like to thank Kazuki Hiroe for pointing to me about the Hecke's result in the case of general number fields [10]. Part of this paper is written during my stay at the Max Planck Institute for Mathematics in 2018. The author is supported by JSPS Overseas Challenge Program for Young Researchers.

\section{References}

[1] T. Asai, On a certain function analogous to $\log |\eta(z)|$, Nagoya Math. J. 40 (1970), 193-211.

[2] H. Bekki, On periodicity of geodesic continued fractions, J. Number Theory 177 (2017), 181-210.

[3] A. Borel, Cohomologie de $\mathrm{SL}_{n}$ et valeurs de fonctions zeta aux points entiers, Ann. Scuola Norm. Sup. Pisa Cl. Sci. (4) 4 (1977), no. 4, 613-636. 
[4] N. Bourbaki, Commutative algebra. Chapters 1-7, Elements of Mathematics (Berlin), SpringerVerlag, Berlin, 1998. Translated from the French, Reprint of the 1989 English translation.

[5] D. Bump and D. Goldfeld, A Kronecker limit formula for cubic fields, Modular forms (Durham, 1983), 1984, pp. 43-49.

[6] A. Costa and E. Friedman, Ratios of regulators in totally real extensions of number fields, J. Number Theory 37 (1991), no. 3, 288-297.

[7] P. Epstein, Zur Theorie allgemeiner Zetafunctionen, Math. Ann. 56 (1903), no. 4, 615-644.

[8] D. Goldfeld, Automorphic forms and L-functions for the group $\operatorname{GL}(n, \mathbf{R})$, Cambridge Studies in Advanced Mathematics, vol. 99, Cambridge University Press, Cambridge, 2006. With an appendix by Kevin A. Broughan.

[9] G. Harder, Period integrals of Eisenstein cohomology classes and special values of some L-functions, Number theory related to Fermat's last theorem (Cambridge, Mass., 1981), 1982, pp. 103-142.

[10] E. Hecke, Über die Kroneckersche Grenzformel für reelle quadratische Körper und die Klassenzahl relativ-Abelscher Körper, Verhandl. Naturforsch. Ges. Basel 28 (1917), 363-372.

[11] K. Hiroe and T. Oda, Hecke-Siegel's pull-back formula for the Epstein zeta function with a harmonic polynomial, J. Number Theory 128 (2008), no. 4, 835-857.

[12] J. Jorgenson and S. Lang, Hilbert-Asai Eisenstein series, regularized products, and heat kernels, Nagoya Math. J. 153 (1999), 155-188.

[13] R. P. Langlands, On the functional equations satisfied by Eisenstein series, Lecture Notes in Mathematics, Vol. 544, Springer-Verlag, Berlin-New York, 1976.

[14] S.-C. Liu and R. Masri, A Kronecker limit formula for totally real fields and arithmetic applications, Res. Number Theory 1 (2015), Art. 8, 20.

[15] P. Sarnak, Class numbers of indefinite binary quadratic forms, J. Number Theory 15 (1982), no. 2, 229-247.

[16] R. Sczech, Eisenstein group cocycles for $\mathrm{GL}_{n}$ and values of L-functions, Invent. Math. 113 (1993), no. $3,581-616$.

[17] C. L. Siegel, Advanced analytic number theory, Second, Tata Institute of Fundamental Research Studies in Mathematics, vol. 9, Tata Institute of Fundamental Research, Bombay, 1980.

[18] S. Yamamoto, Hecke's integral formula for relative quadratic extensions of algebraic number fields, Nagoya Math. J. 189 (2008), 139-154.

[19] H. Yoshida, Absolute CM-periods, Mathematical Surveys and Monographs, vol. 106, American Mathematical Society, Providence, RI, 2003.

Graduate School of Mathematical Sciences, The University of Tokyo, 3-8-1 Komaba, Meguro, Tokyo, 153-8914 Japan

Email: bekki@ms.u-tokyo.ac.jp 